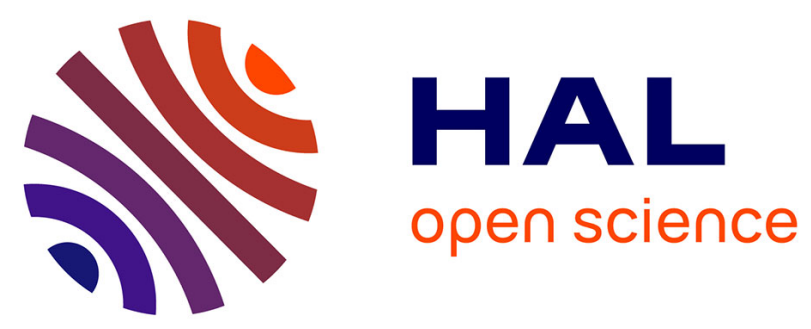

\title{
DEL-sequents for progression
}

Guillaume Aucher

\section{To cite this version:}

Guillaume Aucher. DEL-sequents for progression. Journal of Applied Non-Classical Logics, 2011, 21 (3-4), pp.289-321. 10.3166/jancl.21.289-321 . hal-00674150

\section{HAL Id: hal-00674150 https://inria.hal.science/hal-00674150}

Submitted on 7 Sep 2015

HAL is a multi-disciplinary open access archive for the deposit and dissemination of scientific research documents, whether they are published or not. The documents may come from teaching and research institutions in France or abroad, or from public or private research centers.
L'archive ouverte pluridisciplinaire HAL, est destinée au dépôt et à la diffusion de documents scientifiques de niveau recherche, publiés ou non, émanant des établissements d'enseignement et de recherche français ou étrangers, des laboratoires publics ou privés. 


\title{
DEL-sequents for Progression
}

\author{
Guillaume Aucher \\ INRIA - Université de Rennes 1 \\ Campus Beaulieu \\ 35042 Rennes cedex (France) \\ guillaume.aucher@irisa.fr
}

\begin{abstract}
Dynamic Epistemic Logic (DEL) deals with the representation and the study in a multi-agent setting of knowledge and belief change. It can express in a uniform way epistemic statements about:

(i) what is true about an initial situation

(ii) what is true about an event occurring in this situation

(iii) what is true about the resulting situation after the event has occurred.

We axiomatize within the DEL framework what we can infer about (iii) given (i) and (ii). Given three formulas $\phi, \phi^{\prime}$ and $\phi^{\prime \prime}$ describing respectively (i), (ii) and (iii), we also show how to build a formula $\phi \otimes \phi^{\prime}$ which captures all the information which can be inferred about (iii) from $\phi$ and $\phi^{\prime}$. We show how our results extend to other modal logics than K. In our proofs and definitions, we resort to a large extent to the normal form formulas for modal logic originally introduced by Kit Fine. In a companion paper [Aucher, 2012], we axiomatize what we can infer about (ii) given (i) and (iii), and what we can infer about (i) given (ii) and (iii), and show how to build two formulas $\phi \ominus \phi^{\prime \prime}$ and $\phi^{\prime} \oslash \phi^{\prime \prime}$ which capture respectively all the information which can be inferred about (ii) from $\phi$ and $\phi^{\prime \prime}$, and all the information which can be inferred about (i) from $\phi^{\prime}$ and $\phi^{\prime \prime}$.
\end{abstract}

\section{Introduction}

Dynamic Epistemic Logic (DEL) deals with the representation and the study in a multi-agent setting of knowledge and belief change, and more generally of information change [van Ditmarsch et al., 2007]. The core idea of DEL is to split the task of representing the agents' beliefs into three parts: first, one represents their beliefs about an initial situation; second, one represents their beliefs about an event taking place in this situation; third, one represents the way the agents update their beliefs about the situation after (or during) the occurrence of the event. Consequently, within the logical framework of DEL, one can express uniformly epistemic statements about:

(i) what is true about an initial situation, 
(ii) what is true about an event occurring in this situation,

(iii) what is true about the resulting situation after the event has occurred.

From a logical point of view, this trichotomy begs the following three questions (which were already raised in [Kooi, 2007]). In these questions, $\phi, \phi^{\prime}$ and $\phi^{\prime \prime}$ are three epistemic formulas describing respectively (i), (ii) and (iii).

\section{Question 1:}

1. Given (i) and (ii), what can we infer about (iii): $\phi, \phi^{\prime} \models \phi^{\prime \prime}$ ?

2. How can we build a single formula $\phi \otimes \phi^{\prime}$ which captures all the information which can be inferred about (iii) from $\phi$ and $\phi^{\prime}$ ?

\section{Question 2:}

1. Given (i) and (iii), what can we infer about (ii): $\phi, \phi^{\prime \prime} \models \phi^{\prime}$ ?

2. How can we build a single formula $\phi \otimes \phi^{\prime \prime}$ which captures all the information which can be inferred about (ii) from $\phi$ and $\phi^{\prime \prime}$ ?

\section{Question 3:}

1. Given (ii) and (iii), what can we infer about (i): $\phi^{\prime}, \phi^{\prime \prime} \models \phi$ ?

2. How can we build a single formula $\phi \oslash \phi^{\prime \prime}$ which captures all the information which can be inferred about (i) from $\phi^{\prime}$ and $\phi^{\prime \prime}$ ?

Providing formal tools that answer these questions leads to applications in artificial intelligence and theoretical computer science, and as it turns out, some of them have already been addressed in DEL and other logical formalisms. ${ }^{1}$

Question 1: Progression. Answering the first question leads to the development of tools that can be used by (artificial) agents to compute autonomously their representation of situations as events occur or to reason about the effects of these events. This question has been addressed in the situation calculus, where it is related to the notion of progression [Reiter, 2001]. In the logics of programs, our DEL-sequent $\phi, \phi^{\prime} \models \phi^{\prime \prime}$ correspond to the partial correctness specifications $\{\phi\} \pi\left\{\phi^{\prime \prime}\right\}$ of Hoare's logic [Hoare, 1969] which read as "after every successful execution of program $\pi$ starting from a state where precondition $\phi$ holds, postcondition $\phi^{\prime \prime}$ holds in the final state". Likewise, our formula $\phi \otimes \phi^{\prime}$ corresponds to the strongest post-condition of Propositional Dynamic Logic [Pratt, 1976]. That the product update of DEL is in fact the same as the strongest post-condition has been elaborated on and proved in an algebraic setting in [Baltag et al., 2005]. A sequent calculus is also provided in this algebraic setting.

\footnotetext{
${ }^{1}$ The question of determining the exact relationship of DEL with other logical formalims has recently started to be investigated. The interested reader can consult [van Benthem, 2011, van Ditmarsch et al., 2009] for its relationship with the situation calculus and [van Benthem et al., 2009a] for its relationship with epistemic temporal logic.
} 
Question 2: Epistemic planning. Answering the second question also leads to applications in artificial intelligence in the area of epistemic planning: (artificial) agents often need to determine autonomously which actions they need to perform in order to achieve a given epistemic goal. This second question is also related to the notion of explanation and has been dealt with in the event calculus of [Shanahan, 1997] for instance, where it is shown that planning problems can be handled via abduction (using logic programming). In computer science, this second question is also related to the synthesis problem raised by Church in its full generality [Church, 1957]. He asked whether, given a desired relation between a set of inputs and a set of outputs, we can construct a function that produces the desired outputs from arbitrary inputs. This problem has been declined as the problem of program synthesis: given a specification, can we construct a program that is guaranteed to satisfy this specification? It was extensively studied in the 1980s and 1990s for temporal logic specifications. The synthesis problem is more challenging when the input is incomplete [Kupferman \& Vardi, 1999]. Open (reactive) environments can be a reason of incompleteness of the input, and epistemic logic is a natural formalism to resort to model such situations, as argued in [Halpern \& Moses, 1990]. For single-agent temporal epistemic logic, this synthesis problem has been solved in [van der Meyden \& Vardi, 1998]. However, this problem has not been addressed so far within the DEL approach, although its methodology and formal setting lend itself rather naturally to address it.

Question 3: Regression. Answering the third question is related to the notion of regression introduced in the situation calculus [Reiter, 2001]. This technique is used to determine whether a statement holds after a sequence of events (called the projection problem) by reducing (regressing) this statement about the resulting situation to a statement about the initial situation. In DEL, regression corresponds to the classical reduction method used to prove completeness of an axiomatization: a formula with dynamic operator(s) is 'reduced' equivalently to a formula without dynamic operator by pushing the dynamic operator through the logical connectives, performing some kind of regression of the initial formula with dynamic operator. In the companion paper [Aucher, 2012], our inductive definition of the regression of $\phi^{\prime \prime}$ by $\phi^{\prime}$, i.e. $\phi^{\prime} \oslash \phi^{\prime \prime}$, is based on the reduction axioms of DEL [Baltag \& Moss, 2004]. Note that in Propositional Dynamic Logic, $\neg\left(\phi \oslash \neg \phi^{\prime \prime}\right)$ also corresponds to the weakest precondition.

In this paper, we will answer the first question within the DEL framework. The second and third questions are dealt with in a companion paper [Aucher, 2012]. In a third paper [Aucher et al., 2011], we provide a tableau method to decide whether an inference of one of the three kinds above holds and show that this decision problem is NEXPTIME-complete. The tableau method is also implemented in LOTRECscheme.

From a conceptual perspective, axiomatizing the first inference relation $\phi, \phi^{\prime} \models \phi^{\prime \prime}$ leads to a natural characterization of the notion of (belief) update. This notion can therefore be studied rigorously and systematically, as it has been done in the restricted setting of a single agent in the theory of belief revision of [Alchourrón et al., 1985] 
and the theory of belief update of [Katsuno \& Mendelzon, 1992]. In particular, this axiomatic/postulational approach permits a natural comparison, analysis and introduction of new revision and update operations. In DEL, [van Benthem, 2007] proposes another approach to analyse updates. The idea is to apply the same technique used in modal logic for modal frame correspondance to the study of the modal operator of update. Dynamic axioms can characterize classes of operations on models in the way that classical modal axioms characterize classes of frames. This approach is at the same time less restrictive than ours, but also more abstract. Less restrictive in the sense that the language used can combine within a single formula expressions dealing with (i), (ii) and (iii) altogether, unlike our DEL-sequents where these expressions are clearly separated. More abstract in the sense that what is really studied is the inference relation from (i) to (iii), the second item (ii) being somehow abstracted away. However, in its current form, this approach cannot account explicitly for specific classes of updates induced by classes of event models as the ones typically used in DEL, simply because the language cannot refer to classes of event models.

The paper is organized as follows. In Section 2, we introduce our logical formalism and show how one can naturally express epistemic statements about (i), (ii) and (iii) within this framework. In Section 3, we introduce some mathematical objects needed in the subsequent proofs, namely normal form formulas and the notion of refinement. We also characterize this notion of refinement syntactically by means of our normal form formulas. In Section 4, we provide two equivalent sequent calculi which axiomatize the inference relation of Question 1) a), both for epistemic and ontic events. In Section 5 , we propose a constructive definition of the formula $\phi \otimes \phi^{\prime}$ of Question 1) b). In Section 6, we show how our results extend to other modal logics than $\mathrm{K}$, and in Section 7 we give some examples of derivations of valid inferences in our calculi. We end the paper in Section 8 by some concluding remarks.

\section{Dynamic Epistemic Logic}

Following the DEL methodology described above, we split the exposition of our logical formalism into three subsections. In the rest of the paper, Agt is a finite set of agents and $\Phi$ is a set of propositional letters called atomic facts.

\subsection{Representation of the initial situation: $\mathcal{L}$-model}

A (pointed) $\mathcal{L}$-model $(M, w)$ represents how the actual world represented by $w$ is perceived by the agents. Atomic facts are used to state properties of this actual world.

Definition 1 ( $\mathcal{L}$-model). A $\mathcal{L}$-model is a tuple $M=(W, R, V)$ where $W$ is a non-empty set of possible worlds, $R: A g t \rightarrow 2^{W \times W}$ is a function assigning to each agent $j \in A g t$ an accessibility relation on $W$, and $V: \Phi \rightarrow 2^{W}$ is a function called a valuation assigning to each propositional letter of $\Phi$ a subset of $W$.

We write $w \in M$ for $w \in W$, and $(M, w)$ is called a pointed $\mathcal{L}$-model. If $w, v \in W$, we write $w R_{j} v$ for $R(j)(w, v)$ and $R_{j}(w)$ for $\left\{v \in W \mid w R_{j} v\right\}$. 
Intuitively, in the definition above, $v \in R_{j}(w)$ means that in world $w$ agent $j$ considers world $v$ as being possibly the world $w$.

Now, we define the epistemic language $\mathcal{L}$ which can be used to describe and state properties of $\mathcal{L}$-models. In particular, the formula $B_{j} \phi$ reads as "agent $j$ Believes $\phi$ ". Its truth conditions are defined in such a way that $B_{j} \phi$ holds in a possible world when $\phi$ holds in all the worlds agent $j$ considers possible. Dually, the formula $\left\langle B_{j}\right\rangle \phi$ reads as "agent $j$ considers possible that $\phi$ holds".

Definition 2 (Language $\mathcal{L}$ ). We define the language $\mathcal{L}$ inductively as follows:

$$
\mathcal{L}: \phi::=p|\neg \phi| \phi \wedge \phi \mid B_{j} \phi
$$

where $p$ ranges over $\Phi$ and $j$ over Agt. The formula $\phi \vee \psi$ is an abbreviation for $\neg(\neg \phi \wedge \neg \psi) ; \phi \rightarrow \psi$ is an abbreviation for $\neg \phi \vee \psi$; and $\left\langle B_{j}\right\rangle \phi$ is an abbreviation for $\neg B_{j} \neg \phi .{ }^{2}$ If $\phi \in \mathcal{L}$, then we note $P(\phi)$ the set of atomic events appearing in $\phi$.

Let $M$ be a $\mathcal{L}$-model, $w \in M$ and $\phi \in \mathcal{L}$. The satisfaction relation $M, w \vDash \phi$ is defined inductively as follows:

$$
\begin{array}{lll}
M, w \models p & \text { iff } & w \in V(p) \\
M, w=\neg \phi & \text { iff } & \text { not } M, w \models \phi \\
M, w \models \phi \wedge \psi & \text { iff } & M, w \models \phi \text { and } M, w \models \psi \\
M, w=B_{j} \phi & \text { iff } & \text { for all } v \in R_{j}(w), M, v \models \phi
\end{array}
$$

We write $M \vDash \phi$ when $M, w \vDash \phi$ for all $w \in M$, and $\vDash \phi$ when $M \models \phi$ for all $\mathcal{L}$-model $M$.

Example 1. Assume that agents A, B and C play a card game with three cards: a white one, a red one and a blue one. Each of them has a single card but they do not know the cards of the other players. At each step of the game, some of the players show their/her/his card to another player or to both other players, either privately or publicly. We want to study and represent the dynamics of the agents' beliefs in this game. The initial situation is represented by the pointed $\mathcal{L}$-model $(M, w)$ of Figure 1 . In this example, $\Phi=\left\{r_{j}, b_{j}, w_{j} \mid j \in\{A, B, C\}\right\}$ where $r_{j}$ stands for 'agent $j$ has the $r$ ed card', $b_{j}$ stands for 'agent $\mathrm{j}$ has the $b$ lue card' and $w_{j}$ stands for 'agent $j$ has the white card'. The boxed possible world corresponds to the actual world. The propositional letters not mentioned in the possible worlds do not hold in these possible worlds. The accessibility relations are represented by arrows indexed by agents between possible worlds. Reflexive arrows are omitted in the figure, which means that for all worlds $v \in M$ and all agents $j \in\{A, B, C\}, v \in R_{j}(v)$. In this model, we have for example the following statement: $M, w \vDash\left(w_{B} \wedge \neg B_{A} w_{B}\right) \wedge B_{C} \neg B_{A} w_{B}$. It states that player A does not 'know' that player $B$ has the white card and player $C$ 'knows' it.

Theorem 1 (Soundness and completeness of K). [Blackburn et al., 2001] The logic K is defined by the following axiom schemata and inference rules:

$$
\begin{array}{ll}
\text { (Propositional) } & \text { All propositional axiom schemata and inference rules } \\
\text { ( } B_{j} \text {-distribution) } & \vdash B_{j}(\phi \rightarrow \psi) \rightarrow\left(B_{j} \phi \rightarrow B_{j} \psi\right) \\
\text { ( } B_{j} \text {-necessitation) } & \text { If } \vdash \phi \text { then } \vdash B_{j} \phi
\end{array}
$$

\footnotetext{
${ }^{2}$ The degree $\operatorname{deg}(\phi)$ of a formula $\phi \in \mathcal{L}$ is defined inductively as follows: $\operatorname{deg}(p)=0, \operatorname{deg}(\neg \phi)=$ $\operatorname{deg}(\phi), \operatorname{deg}(\phi \wedge \psi)=\max \{\operatorname{deg}(\phi), \operatorname{deg}(\psi)\}, \operatorname{deg}\left(B_{j} \phi\right)=1+\operatorname{deg}(\phi)$. We define similarly the degree $\operatorname{deg}\left(\phi^{\prime}\right)$ of a formula $\phi^{\prime}$ from the language $\mathcal{L}^{\prime}$ of Definition 5 .
} 


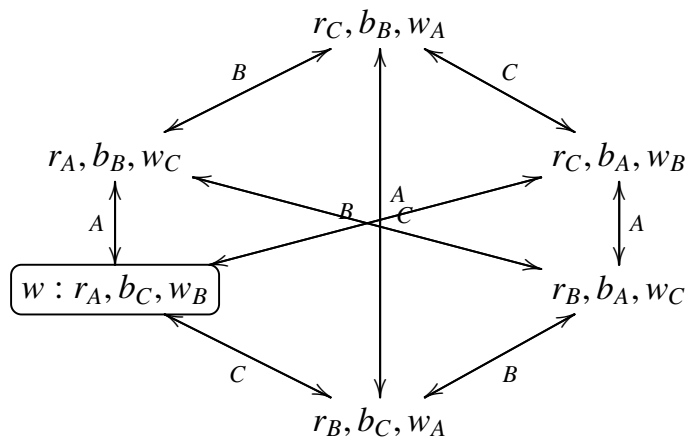

Figure 1: Cards Example

A formula $\phi \in \mathcal{L}$ is a K-theorem, written $\phi \in K$ or $\vdash \phi$, when $\phi$ can be derived by successively applying (some of) the inference rules on (some of) the axioms. We say that $\phi$ is $\mathrm{K}$-inconsistent when $\neg \phi$ is derivable in $\mathrm{K}$, and $\mathrm{K}$-consistent otherwise. Then, for all $\phi \in \mathcal{L}, \vdash \phi$ implies that $\models \phi$ (soundness), and $\models \phi$ implies that $\vdash \phi$ (completeness).

\subsection{Representation of the event: $\mathcal{L}^{\prime}$-model}

The propositional letters $p^{\prime}$ describing events are called atomic events and range over an infinite set $\Phi^{\prime}$. To each atomic event $p^{\prime}$, we assign a formula $\operatorname{Pre}\left(p^{\prime}\right)$ of the language $\mathcal{L}$, which is called the precondition of $p^{\prime}$. This precondition corresponds to the property that should be true at any world $w$ of a $\mathcal{L}$-model so that the atomic event $p^{\prime}$ can 'physically' occur in this world $w$. Note that the definition below constrains indirectly the definition of the infinite set $\Phi^{\prime}$.

Definition 3 (Precondition function). A precondition function Pre : $\Phi^{\prime} \rightarrow \mathcal{L}$ is a function which assigns to each propositional letter $p^{\prime}$ a formula of $\mathcal{L}$ such that for all $\psi \in \mathcal{L}$, there is $p^{\prime} \in \Phi^{\prime}$ such that $\operatorname{Pre}\left(p^{\prime}\right)=\psi$.

A pointed $\mathcal{L}^{\prime}$-model $\left(M^{\prime}, w^{\prime}\right)$ represents how the actual event represented by $w^{\prime}$ is perceived by the agents.

Definition 4 ( $\mathcal{L}^{\prime}$-model). A $\mathcal{L}^{\prime}$-model is a tuple $M^{\prime}=\left(W^{\prime}, R^{\prime}, V^{\prime}\right)$ where $W^{\prime}$ is a nonempty set of possible events, $R^{\prime}: A g t \rightarrow 2^{W^{\prime} \times W^{\prime}}$ is a function assigning to each agent $j \in A g t$ an accessibility relation on $W^{\prime}$, and $V^{\prime}: \Phi^{\prime} \rightarrow 2^{W^{\prime}}$ is a function called a valuation assigning to each propositional letter of $\Phi^{\prime}$ a subset of $W^{\prime}$ such that

for all $w^{\prime} \in W^{\prime}$, there is at most one $p^{\prime}$ such that $w^{\prime} \in V\left(p^{\prime}\right)$. (Exclusivity)

We write $w^{\prime} \in M^{\prime}$ for $w^{\prime} \in W^{\prime}$, and $\left(M^{\prime}, w^{\prime}\right)$ is called a pointed $\mathcal{L}^{\prime}$-model. If $w^{\prime}, v^{\prime} \in W^{\prime}$, we write $w^{\prime} R_{j}^{\prime} v^{\prime}$ for $R^{\prime}(j)\left(w^{\prime}, v^{\prime}\right)$ and $R_{j}^{\prime}\left(w^{\prime}\right)$ for $\left\{v^{\prime} \in W^{\prime} \mid w^{\prime} R_{j}^{\prime} v^{\prime}\right\}$.

Intuitively, $v^{\prime} \in R_{j}\left(w^{\prime}\right)$ means that while the possible event represented by $w^{\prime}$ is occurring, agent $j$ considers possible that the possible event represented by $v^{\prime}$ is actually 
occurring. The condition (Exclusivity) expresses in our framework the fact that a single precondition is assigned to each possible event, as in the standard BMS framework of [Baltag \& Moss, 2004].

In fact, the definition of a $\mathcal{L}^{\prime}$-model is equivalent to the definition of an action signature in the logical formalism of [Baltag \& Moss, 2004]. Indeed, an action signature is a tuple $\Sigma=\left(W^{\prime}, R^{\prime},\left(w_{1}^{\prime}, \ldots, w_{n}^{\prime}\right)\right)$ where: 1) $W^{\prime}$ is a non-empty and finite set of action types (possible events are called "action types" in the BMS formalism), 2) $R^{\prime}: A g t \rightarrow 2^{W^{\prime} \times W^{\prime}}$ is a function assigning to each agent $j \in A g t$ an accessibility relation on $W^{\prime}$, and 3) $\left\{w_{1}^{\prime}, \ldots, w_{n}^{\prime}\right\}$ is a subset of $W^{\prime}$ such that for all $i, j \in\{1, \ldots, n\}$, if $i \neq j$ then $w_{i}^{\prime} \neq w_{j}^{\prime}$. If we consider an action signature $\Sigma=\left(W^{\prime}, R^{\prime},\left(w_{1}^{\prime}, \ldots, w_{n}^{\prime}\right)\right)$ together with a set of formulas $\phi_{1}, \ldots, \phi_{n} \in \mathcal{L}$, then we can get back an $\mathcal{L}^{\prime}$-model: the $\mathcal{L}^{\prime}$-model associated to $\left(\Sigma, \phi_{1}, \ldots, \phi_{n}\right)$ is the tuple $M^{\prime}=\left(W^{\prime}, R^{\prime}, V^{\prime}\right)$ where for all $p^{\prime} \in \Phi^{\prime}, V^{\prime}\left(p^{\prime}\right)$ is equal to $\left\{w_{i}^{\prime}\right\}$ if $\operatorname{Pre}\left(p^{\prime}\right)=\phi_{i}$ for some $i$, and equal to the empty set otherwise.

Just as we defined a language $\mathcal{L}$ for epistemic models, we also define a language $\mathcal{L}^{\prime}$ for $\mathcal{L}^{\prime}$-models whose truth conditions are identical to the ones of the language $\mathcal{L}$. This language was already introduced in [Baltag et al., 1999]. In the sequel, formulas of $\mathcal{L}^{\prime}$ will always be indexed by the quotation mark', unlike formulas of $\mathcal{L}$.

Definition 5 (Language $\mathcal{L}^{\prime}$ ). We define the language $\mathcal{L}^{\prime}$ inductively as follows:

$$
\mathcal{L}^{\prime}: \phi^{\prime}::=p^{\prime}\left|\neg \phi^{\prime}\right| \phi^{\prime} \wedge \phi^{\prime} \mid B_{j} \phi^{\prime}
$$

where $p^{\prime}$ ranges over $\Phi^{\prime}$ and $j$ over Agt. The formula $\phi^{\prime} \vee \psi^{\prime}$ is an abbreviation for $\neg\left(\neg \phi^{\prime} \wedge \neg \psi^{\prime}\right) ; \phi^{\prime} \rightarrow \psi^{\prime}$ is an abbreviation for $\neg \phi^{\prime} \vee \psi^{\prime}$; and $\left\langle B_{j}\right\rangle \phi^{\prime}$ is an abbreviation for $\neg B_{j} \neg \phi^{\prime}$. If $\phi^{\prime} \in \mathcal{L}^{\prime}$, then we note $P^{\prime}\left(\phi^{\prime}\right)$ the set of atomic events appearing in $\phi^{\prime}$.

Let $M^{\prime}$ be a $\mathcal{L}^{\prime}$-model, $w^{\prime} \in M^{\prime}$ and $\phi^{\prime} \in \mathcal{L}^{\prime}$. The satisfaction relation $M^{\prime}, w^{\prime} \vDash \phi^{\prime}$ is defined inductively as follows:

$$
\begin{array}{lll}
M^{\prime}, w^{\prime} \models p^{\prime} & \text { iff } & w^{\prime} \in V^{\prime}\left(p^{\prime}\right) \\
M^{\prime}, w^{\prime} \models \neg \phi^{\prime} & \text { iff } & \text { not } M^{\prime}, w^{\prime} \models \phi^{\prime} \\
M^{\prime}, w^{\prime} \models \phi^{\prime} \wedge \psi^{\prime} & \text { iff } & M^{\prime}, w^{\prime} \models \phi^{\prime} \text { and } M^{\prime}, w^{\prime} \models \psi^{\prime} \\
M^{\prime}, w^{\prime} \vDash B_{j} \phi^{\prime} & \text { iff } & \text { for all } v^{\prime} \in R_{j}^{\prime}\left(w^{\prime}\right), M^{\prime}, v^{\prime} \models \phi^{\prime} .
\end{array}
$$

We write $M^{\prime} \vDash \phi^{\prime}$ when $M^{\prime}, w^{\prime} \vDash \phi^{\prime}$ for all $w^{\prime} \in M^{\prime}$, and $\models \phi^{\prime}$ when $M^{\prime} \vDash \phi^{\prime}$ for all $\mathcal{L}^{\prime}$-model $M^{\prime}$.

Example 2. Let us resume Example 1 and assume that players A and B show their card to each other. As it turns out, $\mathrm{C}$ noticed that $\mathrm{A}$ showed her card to B but did not notice that B did so to A. Players A and B know this. This event is represented in the $\mathcal{L}^{\prime}$-model $\left(M^{\prime}, w^{\prime}\right)$ of Figure 2 . The boxed possible event $w^{\prime}$ corresponds to the actual event. The atomic event $p^{\prime}$ stands for 'player A shows her red card', $q^{\prime}$ stands for the atomic event 'player A shows her white card' and $r$ ' stands for the atomic event 'players A and B show their red and $w$ hite cards respectively to each other'. The precondition $\operatorname{Pre}\left(p^{\prime}\right)$ of $p^{\prime}$ is $r_{A}$, the precondition $\operatorname{Pre}\left(q^{\prime}\right)$ of $q^{\prime}$ is $w_{A}$, and the precondition $\operatorname{Pre}\left(r^{\prime}\right)$ of $r^{\prime}$ is $r_{A} \wedge w_{B}$. We mention in the possible events of the $\mathcal{L}^{\prime}$-model only the atomic events 


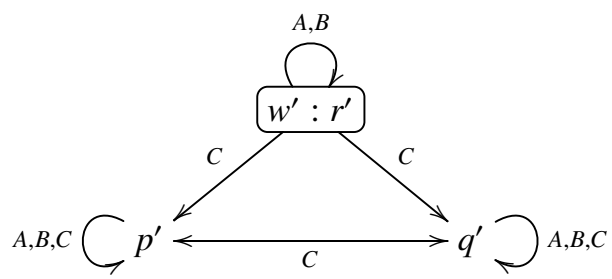

Figure 2: Players A and B show their cards to each other in front of player C

that hold in these possible events. The following statement holds in our example:

$$
\begin{aligned}
M^{\prime}, w^{\prime}=r^{\prime} & \wedge\left(\left\langle B_{A}\right\rangle r^{\prime} \wedge B_{A} r^{\prime}\right) \wedge\left(\left\langle B_{B}\right\rangle r^{\prime} \wedge B_{B} r^{\prime}\right) \\
& \wedge\left(\left\langle B_{C}\right\rangle p^{\prime} \wedge\left\langle B_{C}\right\rangle q^{\prime} \wedge B_{C}\left(p^{\prime} \vee q^{\prime}\right)\right)
\end{aligned}
$$

It states that players A and B show their cards to each other, players A and B 'know' this and consider it possible, while player $\mathrm{C}$ considers possible that player A shows her white card and also considers possible that player A shows her red card, since he does not know her card. In fact, that is all that player $\mathrm{C}$ considers possible since he believes that either player A shows her red card or her white card.

Remark 1. Note that the ontological nature of events (such as 'looking at the card' versus 'being shown the card') is irrelevant as far as change of beliefs about the world is concerned, because the epistemic change potential of epistemic events is fully determined by their preconditions and their epistemic indistinguishability. However, when it comes to account for change of beliefs about events themselves, their ontological nature does play a role, because this ontological nature may be the subject of preconditions of some other 'meta'-events. The change of beliefs about events due to the occurrence of some other 'meta'-events is studied within the DEL framework in [Aucher, 2009].

Now, we introduce the notion of $P^{\prime}$-complete models which will play a technical role in the axiomatization of our DEL-sequents in the next sections.

Definition 6 ( $P^{\prime}$-complete $\mathcal{L}^{\prime}$-model). Let $P^{\prime}$ be a finite subset of $\Phi^{\prime}$. A $P^{\prime}$-complete $\mathcal{L}^{\prime}$-model is a $\mathcal{L}^{\prime}$-model $M^{\prime}$ such that

for all $w^{\prime} \in M^{\prime}$, there is a unique $p^{\prime} \in P^{\prime}$ such that $w^{\prime} \in V^{\prime}\left(p^{\prime}\right) . \quad\left(P^{\prime}\right.$-complete $)$

A complete $\mathcal{L}^{\prime}$-model is a $\Phi^{\prime}$-complete $\mathcal{L}^{\prime}$-model $M^{\prime}$.

Theorem 2 (Soundness and completeness of $\mathrm{K}^{\prime}$ and $\mathrm{K}^{\mathrm{P}^{\prime}}$ ). The logic $\mathrm{K}^{\prime}$ is defined by the following axiom schemata and inference rules:

(Propositional) All propositional axiom schemata and inference rules

(B $B_{j}$-distribution) $\quad \vdash^{\prime} B_{j}\left(\phi^{\prime} \rightarrow \psi^{\prime}\right) \rightarrow\left(B_{j} \phi^{\prime} \rightarrow B_{j} \psi^{\prime}\right)$

$\left(B_{j}\right.$-necessitation) If $\vdash^{\prime} \phi^{\prime}$ then $\vdash^{\prime} B_{j} \phi^{\prime}$

(Exclusivity) $\quad r^{\prime} p^{\prime} \rightarrow \neg q^{\prime} \quad$ for all $p^{\prime} \neq q^{\prime}$ 
Let $P^{\prime}$ be a finite subset of $\Phi^{\prime}$. The logic $K^{P^{\prime}}$ is defined by adding to the logic $K^{\prime}$ the following axiom:

$$
\text { ( } \left.P^{\prime} \text {-Complete }\right) \quad \quad^{\prime} \bigvee_{p^{\prime} \in P^{\prime}} p^{\prime}
$$

We say that a formula $\phi^{\prime} \in \mathcal{L}^{\prime}$ is a $K^{\prime}$-theorem, written $\phi^{\prime} \in K^{\prime}$ or $r^{\prime} \phi^{\prime}$, when $\phi^{\prime}$ can be derived by successively applying (some of) the inference rules on (some of) the axioms of $K^{\prime}$. We say that $\phi^{\prime}$ is $\mathrm{K}^{\prime}$-inconsistent when $\neg \phi^{\prime}$ is derivable in $K^{\prime}$, and $\mathrm{K}^{\prime}$-consistent otherwise. Then, for all $\phi^{\prime} \in \mathcal{L}^{\prime}, r^{\prime} \phi^{\prime}$ implies that $\models^{\prime} \phi^{\prime}$ (soundness) and $\models^{\prime} \phi^{\prime}$ implies $r^{\prime} \phi^{\prime}$ (completeness). Similar definitions and results hold for $K^{P^{\prime}}$.

Proof (Sketch). Soundness is standard. Completeness can easily be proved by adapting the canonical model construction for $\mathrm{K}$ given in [Blackburn et al., 2001].

\subsection{Update of the initial situation by the event: product update}

The precondition function of Definition 3 induces a precondition function for $\mathcal{L}^{\prime}$ models, which assigns to each possible event $w^{\prime}$ of a $\mathcal{L}^{\prime}$-model a formula of $\mathcal{L}$. This formula corresponds to the property that should be true at any world $w$ of a $\mathcal{L}$-model so that the possible event $w^{\prime}$ can 'physically' occur in the world $w$.

Definition 7 (Precondition function of a $\mathcal{L}^{\prime}$-model). Let $M^{\prime}=\left(W^{\prime}, R^{\prime}, V^{\prime}\right)$ be a $\mathcal{L}^{\prime}$ model. The precondition function of $M^{\prime}$ is the function Pre : $W^{\prime} \rightarrow \mathcal{L}$ defined as follows:

$$
\operatorname{Pre}\left(w^{\prime}\right)= \begin{cases}\operatorname{Pre}\left(p^{\prime}\right) & \text { if there is } p^{\prime} \text { such that } M^{\prime}, w^{\prime} \vDash p^{\prime} \\ \top & \text { otherwise. }\end{cases}
$$

where $\mathrm{T}$ is any $\mathrm{K}$-theorem.

We then redefine equivalently in our setting the BMS product update of [Baltag $\&$ Moss, 2004]. This product update takes as argument a pointed $\mathcal{L}$-model $(M, w)$ and a pointed $\mathcal{L}^{\prime}$ model $\left(M^{\prime}, w^{\prime}\right)$ representing respectively how an initial situation is perceived by the agents and how an event occurring in this situation is perceived by them, and yields a new pointed $\mathcal{L}$-model $(M, w) \otimes\left(M^{\prime}, w^{\prime}\right)$ representing how the new situation is perceived by the agents after the occurrence of the event.

Definition 8 (Product update). Let $(M, w)=(W, R, V, w)$ be a pointed $\mathcal{L}$-model and $\left(M^{\prime}, w^{\prime}\right)=\left(W^{\prime}, R^{\prime}, V^{\prime}, w^{\prime}\right)$ be a pointed $\mathcal{L}^{\prime}$-model such that $M, w \vDash \operatorname{Pre}\left(w^{\prime}\right)$. The product update of $(M, w)$ and $\left(M^{\prime}, w^{\prime}\right)$ is the pointed $\mathcal{L}$-model $(M, w) \otimes\left(M^{\prime}, w^{\prime}\right)=$ $\left(W^{\otimes}, R^{\otimes}, V^{\otimes},\left(w, w^{\prime}\right)\right)$ defined as follows:

$$
\begin{aligned}
W^{\otimes} & =\left\{\left(v, v^{\prime}\right) \in W \times W^{\prime} \mid M, v \models \operatorname{Pr} e\left(v^{\prime}\right)\right\} \\
R_{j}^{\otimes}\left(v, v^{\prime}\right) & =\left\{\left(u, u^{\prime}\right) \in W^{\otimes} \mid u \in R_{j}(v) \text { and } u^{\prime} \in R_{j}^{\prime}\left(v^{\prime}\right)\right\} \\
V^{\otimes}(p) & =\left\{\left(v, v^{\prime}\right) \in W^{\otimes} \mid M, v \vDash p\right\}
\end{aligned}
$$




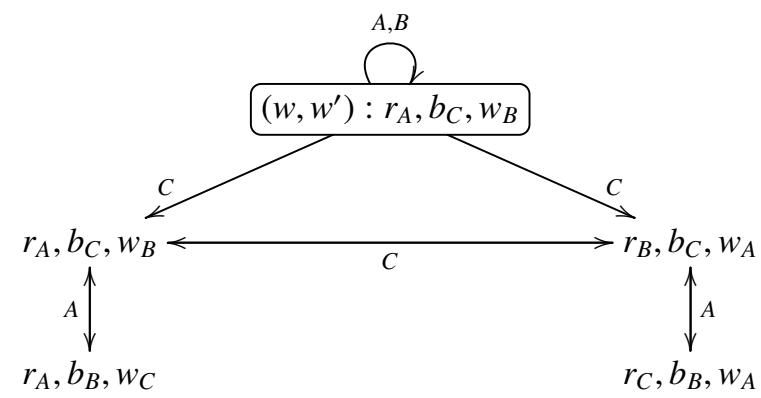

Figure 3: Situation after the update of the situation represented in Figure 1 by the event represented in Figure 2

Example 3. As a result of the event described in Example 2, the agents update their beliefs. We get the situation represented in the $\mathcal{L}$-model $(M, w) \otimes\left(M^{\prime}, w^{\prime}\right)$ of Figure ??. In this model, we have for example the following statement:

$$
(M, w) \otimes\left(M^{\prime}, w^{\prime}\right) \vDash\left(w_{B} \wedge B_{A} w_{B}\right) \wedge B_{C} \neg B_{A} w_{B} .
$$

It states that player A 'knows' that player B has the white card but player C believes that it is not the case.

\section{Mathematical Intermezzo}

In this section, we introduce some mathematical objects that will play a central role from a technical point of view in the sequel.

\subsection{Kit Fine's formulas}

We will resort in our proofs to particular kinds of modal formulas which capture the structure of epistemic models (modulo bisimulation) up to a given modal depth. These formulas were defined in [Moss, 2007] and are very similar to the normal form formulas for modal logic which were originally introduced in [Fine, 1975]. In Section 3.1.1, we introduce them for the logic $\mathrm{K}$. We will adapt these definitions for the logics $\mathrm{K}^{\prime}$ and $\mathrm{K}^{\mathrm{P}^{\prime}}$ in Section 3.1.2. Note that a similar work has been done in [Moss, 2007] for other logics and languages, notably for the modal language with the 'star' operator.

\subsubsection{Kit Fine's formulas for $K$}

A Kit Fine formula $\delta_{n+1}$ provides a complete syntactic representation of a pointed $\mathcal{L}$ model up to modal depth $n+1$. So, intuitively, if we view a Kit Fine formula $\delta_{n+1}$ of $S_{n+1}^{P}$ as the syntactic representation up to modal depth $n+1$ of a possible world $w$ where it holds, a formula $\delta_{n}$ of $S_{n}^{j}$ can also be viewed as a syntactic representation up to 
modal depth $n$ of a possible world accessible by $R_{j}$ from $w$. This justifies our notations in Equation 7.

Definition 9 (Sets $S_{n}^{P}$ ). [Moss, 2007] Let $P$ be a finite subset of $\Phi$. We define inductively the sets $S_{n}^{P}$ for $n \in \mathbb{N}$ as follows:

$$
\begin{aligned}
S_{0}^{P} & =\left\{\bigwedge_{p \in S_{0}} p \wedge \bigwedge_{p \notin S_{0}} \neg p \mid S_{0} \subseteq P\right\} \\
S_{n+1}^{P} & =\left\{\delta_{0} \wedge \bigwedge_{j \in A g t}\left(\bigwedge_{\delta_{n} \in S_{n}^{j}}\left\langle B_{j}\right\rangle \delta_{n} \wedge B_{j}\left(\bigvee_{\delta_{n} \in S_{n}^{j}} \delta_{n}\right)\right) \mid \delta_{0} \in S_{0}^{P}, S_{n}^{j} \subseteq S_{n}^{P}\right\} .
\end{aligned}
$$

A formula of $\delta \in S_{n}^{P}$ for some $n>0$ will often be written as follows:

$$
\delta=\delta_{0} \wedge \bigwedge_{j \in A g t}\left(\bigwedge_{\gamma \in R_{j}(\delta)}\left\langle B_{j}\right\rangle \gamma \wedge B_{j}\left(\bigvee_{\gamma \in R_{j}(\delta)} \gamma\right)\right)
$$

The following proposition not only tells us that a formula $\delta_{n}$ completely characterizes the structure up to modal depth $n$ of any pointed epistemic model where it holds (first item), but also that the structure of any epistemic model up to modal depth $n$ can be characterized by such a formula $\delta_{n}$ (second item). If $(M, w)$ is a pointed $\mathcal{L}$-model, then $\delta_{n}(w)$ will denote the unique element of $S_{n}^{P}$ such that $M, w \vDash \delta_{n}(w)$.

Proposition 3. [Moss, 2007] Let $n \in \mathbb{N}$ and let $P$ be a finite set of propositional letters. Let $\phi \in \mathcal{L}$ be such that $\operatorname{deg}(\phi) \leq n$ and such that $P(\phi) \subseteq P$.

1. For all $\delta_{n} \in S_{n}^{P}$, either $\delta_{n} \rightarrow \phi \in K$ or $\delta_{n} \rightarrow \neg \phi \in K$.

2. $\bigvee_{\delta_{n} \in S_{n}^{P}} \delta_{n} \in K$.

The following corollary will play an important role in the sequel. It states that any formula (of degree $n$ ) can be reduced to a disjunction of $\delta_{n}$ s. This explains why these formulas are called normal form formulas. The decomposition of a formula $\phi$ into $\delta$ s somehow captures completely and syntactically the relevant structure of the set of pointed $\mathcal{L}$-models which make $\phi$ true: each $\delta$ can be seen as a syntactic description of the modal structure (up to depth $n$ and modulo bisimulation) of a pointed $\mathcal{L}$-model which makes $\phi$ true.

Corollary 1. Let $n \in \mathbb{N}$ and let $P$ be a finite subset of $\Phi$. Let $\phi \in \mathcal{L}$ be such that $\operatorname{deg}(\phi) \leq n$ and $P(\phi) \subseteq P$. Then, there is $S \subseteq S_{n}^{P}$ (possibly empty) such that $\phi \leftrightarrow$ $\bigvee_{\delta \in S} \delta \in K$

Proof. Let $\delta_{n}^{1}, \ldots, \delta_{n}^{k}$ be the formulas of $S_{n}^{P}$ such that $\delta_{n}^{i} \rightarrow \phi \in \mathrm{K}$ for all $i \in\{1, \ldots, k\}$. Then $\delta_{n}^{1} \vee \ldots \vee \delta_{n}^{k} \rightarrow \phi \in \mathrm{K}$. Then, by Proposition 3, for all $\delta_{n} \in S_{n}^{P}$ such that 
$\delta_{n} \neq \delta_{n}^{1}, \ldots, \delta_{n} \neq \delta_{n}^{k}$, we have that $\delta_{n} \rightarrow \neg \phi \in \mathrm{K}$. Therefore $\phi \rightarrow \neg \delta_{n} \in \mathrm{K}$ for all $\delta_{n} \in S_{n}^{P}$ such that $\delta_{n} \neq \delta_{n}^{1}, \ldots, \delta_{n} \neq \delta_{n}^{k}$. However, because $\bigvee_{\delta_{n} \in S_{n}^{P}} \delta_{n} \in \mathrm{K}$, we have $\phi \rightarrow \delta_{n}^{1} \vee \ldots \vee \delta_{n}^{k} \in \mathrm{K}$. Finally, $\phi \leftrightarrow\left(\delta_{n}^{1} \vee \ldots \vee \delta_{n}^{k}\right) \in \mathrm{K}$.

\subsubsection{Kit Fine's formulas for $\mathrm{K}^{\prime}$ and $\mathrm{K}^{\mathrm{P}^{\prime}}$}

In this section, we adapt the definitions and propositions of the previous section for the logic $\mathrm{K}^{\prime}$ and $\mathrm{K}^{\mathrm{P}^{\prime}}$. We also define the notion of precondition of a Kit Fine formula for $\mathrm{K}^{\prime}$ and $\mathrm{K}^{\mathrm{P}^{\prime}}$.

Definition 10 (Sets $S_{n}^{P^{\prime}}$ and $E_{n}^{P^{\prime}}$ ). Let $P^{\prime}$ be a finite subset of $\Phi^{\prime}$. We define inductively the sets $S_{n}^{P^{\prime}}$ and $E_{n}^{P^{\prime}}$ for $n \in \mathbb{N}$ as follows:

$$
\begin{aligned}
S_{0}^{P^{\prime}} & =P^{\prime} \cup\left\{\bigwedge_{p^{\prime} \in P^{\prime}} \neg p^{\prime}\right\} \\
S_{n+1}^{P^{\prime}} & =\left\{\delta_{0}^{\prime} \wedge \bigwedge_{j \in A g t}\left(\bigwedge_{\delta_{n}^{\prime} \in S_{n}^{j}}\left\langle B_{j}\right\rangle \delta_{n}^{\prime} \wedge B_{j}\left(\bigvee_{\delta_{n}^{\prime} \in S_{n}^{j}} \delta_{n}^{\prime}\right)\right) \mid \delta_{0}^{\prime} \in S_{0}^{P^{\prime}}, S_{n}^{j} \subseteq S_{n}^{P^{\prime}}\right\} \\
E_{0}^{P^{\prime}} & =P^{\prime} \\
E_{n+1}^{P^{\prime}} & =\left\{\delta_{0}^{\prime} \wedge \bigwedge_{j \in A g t}\left(\bigwedge_{\delta_{n}^{\prime} \in S_{n}^{j}}\left\langle B_{j}\right\rangle \delta_{n} \wedge B_{j}\left(\bigvee_{\delta_{n}^{\prime} \in S_{n}^{j}} \delta_{n}^{\prime}\right)\right) \mid \delta_{0}^{\prime} \in E_{0}^{P^{\prime}}, S_{n}^{j} \subseteq E_{n}^{P^{\prime}}\right\}
\end{aligned}
$$

A formula of $\delta^{\prime} \in S_{n}^{P^{\prime}} \cup E_{n}^{P^{\prime}}$ for some $n>0$ will often be written as follows:

$$
\delta^{\prime}=\delta_{0}^{\prime} \wedge \bigwedge_{j \in A g t}\left(\bigwedge_{\gamma^{\prime} \in R_{j}\left(\delta^{\prime}\right)}\left\langle B_{j}\right\rangle \gamma^{\prime} \wedge B_{j}\left(\bigvee_{\gamma^{\prime} \in R_{j}\left(\delta^{\prime}\right)} \gamma^{\prime}\right)\right)
$$

Note that the only difference between formulas of $S_{n}^{P^{\prime}}$ and $E_{n}^{P^{\prime}}$ lies in the definition of $S_{0}^{P^{\prime}}$ and $E_{0}^{P^{\prime}}$ : the latter only contains formulas of the form $p^{\prime}$ whereas the former also contains the formula $\bigwedge_{p^{\prime} \in P^{\prime}} \neg p^{\prime}$.

Example 4. Formula 1 of Example 2 belongs to $E_{1}^{P^{\prime}}$, with $P^{\prime}=\left\{p^{\prime}, q^{\prime}, r^{\prime}\right\}$.

The following Propositions 4 and 5 are the counterpart of Proposition 3 for the logics $\mathrm{K}^{\prime}$ and $\mathrm{K}^{\mathrm{P}^{\prime}}$, respectively. Both propositions are proved by induction on $n$, similarly to Proposition 3.

Proposition 4. Let $n \in \mathbb{N}$ and let $P^{\prime}$ be a finite subset of $\Phi^{\prime}$. Let $\phi^{\prime} \in \mathcal{L}^{\prime}$ be such that $\operatorname{deg}\left(\phi^{\prime}\right) \leq n$ and such that $P^{\prime}\left(\phi^{\prime}\right) \subseteq P^{\prime}$.

1. For all $\delta_{n}^{\prime} \in S_{n}^{P^{\prime}}$, either $\delta_{n}^{\prime} \rightarrow \phi^{\prime} \in K^{\prime}$ or $\delta_{n}^{\prime} \rightarrow \neg \phi^{\prime} \in K^{\prime}$. 


$$
\text { 2. } \underset{\delta_{n}^{\prime} \in S_{n}^{p^{\prime}}}{\delta_{n}^{\prime} \in K^{\prime}}
$$

Proposition 5. Let $n \in \mathbb{N}$ and let $P^{\prime}$ be a finite subset of $\Phi^{\prime}$. Let $\phi^{\prime} \in \mathcal{L}^{\prime}$ be such that $\operatorname{deg}\left(\phi^{\prime}\right) \leq n$.

1. For all $\delta_{n}^{\prime} \in E_{n}^{P^{\prime}}$, either $\delta_{n}^{\prime} \rightarrow \phi^{\prime} \in K^{P^{\prime}}$ or $\delta_{n}^{\prime} \rightarrow \neg \phi^{\prime} \in K^{P^{\prime}}$.

2. $\bigvee_{\delta_{n}^{\prime} \in E_{n}^{p^{\prime}}} \delta_{n}^{\prime} \in K^{P^{\prime}}$.

The following Corollaries 2 and 3 are the counterpart of Corollary 1 for the logics $\mathrm{K}^{\prime}$ and $\mathrm{K}^{\mathrm{P}^{\prime}}$, respectively. Both corollaries are proved similarly to Corollary 1.

Corollary 2. Let $n \in \mathbb{N}$ and let $P^{\prime}$ be a finite subset of $\Phi^{\prime}$. Let $\phi^{\prime} \in \mathcal{L}^{\prime}$ be such that $\operatorname{deg}\left(\phi^{\prime}\right) \leq n$ and such that $P^{\prime}\left(\phi^{\prime}\right) \subseteq P^{\prime}$. Then, there is $S^{\prime} \subseteq S_{n}^{P^{\prime}}$ (possibly empty) such that $\phi^{\prime} \leftrightarrow \bigvee_{\delta^{\prime} \in S^{\prime}} \delta^{\prime} \in K^{\prime}$.

Corollary 3. Let $n \in \mathbb{N}$ and let $P^{\prime}$ be a finite subset of $\Phi^{\prime}$. Let $\phi^{\prime} \in \mathcal{L}^{\prime}$ be such that $\operatorname{deg}\left(\phi^{\prime}\right) \leq n$. Then, there is $E^{\prime} \subseteq E_{n}^{P^{\prime}}$ (possibly empty) such that $\phi^{\prime} \leftrightarrow \bigvee_{\delta^{\prime} \in E^{\prime}} \delta^{\prime} \in K^{P^{\prime}}$.

The following proposition relates $\mathrm{K}^{\prime}$-consistency with $\mathrm{K}^{\mathrm{P}}$-consistency.

Proposition 6. Let $\phi^{\prime} \in \mathcal{L}^{\prime}$. The formula $\phi^{\prime}$ is $K^{\prime}$-consistent if and only if $\phi^{\prime}$ is $K^{P^{\prime}}$ consistent, and so for any finite subset $P^{\prime}$ of $\Phi^{\prime}$ such that $P^{\prime}\left(\phi^{\prime}\right) \subset P^{\prime}$.

Proof. The right to left direction is trivial. Assume that $\phi^{\prime}$ is $\mathrm{K}^{\prime}$-consistent. Then, by completeness of $\mathrm{K}^{\prime}$, there is a pointed $\mathcal{L}^{\prime}$-model $\left(M^{\prime}, w^{\prime}\right)$ such that $M^{\prime}, w^{\prime} \vDash \phi^{\prime}$. The $\mathcal{L}^{\prime}$-model $\left(M^{\prime}, w^{\prime}\right)$ can easily be modified using an atomic event $q^{\prime} \in P^{\prime}-P^{\prime}\left(\phi^{\prime}\right)$ so that the resulting pointed $\mathcal{L}^{\prime}$-model $\left(M_{P^{\prime}}^{\prime}, w_{P^{\prime}}^{\prime}\right)$ still makes $\phi^{\prime}$ true and is $P^{\prime}$-complete: it suffices to assign the atomic event $q^{\prime}$ to any possible event $w^{\prime}$ of $M^{\prime}$ which does not make any $p^{\prime} \in P^{\prime}\left(\phi^{\prime}\right)$ true. So, by completeness of $\mathrm{K}^{\mathrm{P}^{\prime}}, \phi^{\prime}$ is $\mathrm{K}^{\mathrm{P}^{\prime}}$-consistent, and so for any finite subset $P^{\prime}$ of $\Phi^{\prime}$ such that $P^{\prime}\left(\phi^{\prime}\right) \subset P^{\prime}$.

The precondition of a Kit Fine formula $\delta^{\prime}$ is naturally defined as follows:

Definition 11 (Precondition of $\left.\delta^{\prime}, \operatorname{Pr}\left(\delta^{\prime}\right)\right)$. Let $\delta^{\prime}=\delta_{0}^{\prime} \wedge \bigwedge_{j \in A g t}\left(\bigwedge_{\gamma^{\prime} \in R_{j}\left(\delta^{\prime}\right)}\left\langle B_{j}\right\rangle \gamma^{\prime} \wedge B_{j}\left(\bigvee_{\gamma^{\prime} \in R_{j}\left(\delta^{\prime}\right)} \gamma^{\prime}\right)\right) \in$ $S_{n}^{P^{\prime}} \cup E_{n}^{P^{\prime}}$ for some $n \geq 0$. We define the precondition of $\delta^{\prime}$, written $\operatorname{Pre}\left(\delta^{\prime}\right)$, as follows:

$$
\operatorname{Pre}\left(\delta^{\prime}\right)= \begin{cases}\operatorname{Pre}\left(p^{\prime}\right) & \text { if } \delta_{0}^{\prime}=p^{\prime} \\ \top & \text { otherwise }\end{cases}
$$




\subsection{Refinement}

A refinement is the dual of a simulation, the classical notion of bisimulation being both a refinement and a simulation at the same time [Blackburn et al., 2001].

Definition 12 (Refinement). Let $M_{1}=\left(W_{1}, R_{1}, V_{1}\right)$ and $M_{2}=\left(W_{2}, R_{2}, V_{2}\right)$ be two $\mathcal{L}$ models (or $\mathcal{L}^{\prime}$-models). A non-empty relation $\mathcal{R} \subseteq W_{1} \times W_{2}$ is a refinement iff for all $\left(w_{1}, w_{2}\right) \in \mathcal{R}:$

- for all $p \in \Phi, w_{1} \in V_{1}(p)$ if and only if $w_{2} \in V_{2}(p)$

- for all $v_{2} \in R_{j}\left(w_{2}\right)$, there is $v_{1} \in R_{j}\left(w_{1}\right)$ such that $\left(v_{1}, v_{2}\right) \in \mathcal{R}$.

We say that $\left(M_{2}, w_{2}\right)$ is a refinement of $\left(M_{1}, w_{1}\right)$, which we write $M_{1}, w_{1} \leftarrow M_{2}, w_{2}$, if and only if there exists a refinement relation $\mathcal{R} \subseteq W_{1} \times W_{2}$ such that $\left(w_{1}, w_{2}\right) \in \mathcal{R}$. We say that $\left(M_{2}, w_{2}\right)$ is a refinement up to modal depth $n$ of $\left(M_{1}, w_{1}\right)$, which we write

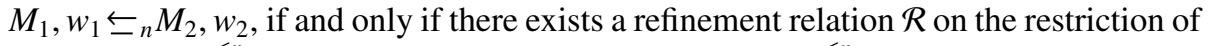
$M_{1}$ to $\left(\bigcup_{j \in A g t} R_{j}\right)^{\leq n}\left(w_{1}\right)$ and the restriction of $M_{2}$ to $\left(\bigcup_{j \in A g t} R_{j}\right)^{\leq n}\left(w_{2}\right)$ such that $\left(w_{1}, w_{2}\right) \in$ $\mathcal{R}^{3}$

The following result will turn out to play a central role in the definition of our notion of progression of $\phi$ by $\phi^{\prime}$ in Section 5 .

Proposition 7. [van Ditmarsch $\mathcal{F}$ French, 2008] Let $M_{1}$ and $M_{2}$ be two finite $\mathcal{L}$-models. $M_{1} \leftarrow M_{2}$ if and only if there is a $\mathcal{L}^{\prime}$-model $M^{\prime}$ such that $M_{1} \otimes M^{\prime}=M_{2}$.

Now, we provide a syntactic characterization of the notion of refinement up to modal depth $n$. Intuitively, the refinement of a $\mathcal{L}$-model is another $\mathcal{L}$-model where some accessibility relations have been removed, modulo bisimulation. This cutting of accessibility relations is reflected in the subset condition of Equation 12.

Definition 13 (Function $\operatorname{Ref}$ ). Let $n \in \mathbb{N}$ and let $P$ be a finite subset of $\Phi$. We define the function $\operatorname{Ref}: S_{n}^{P} \rightarrow 2^{S_{n}^{P}}$ inductively as follows:

$n=0$ : for all $\delta_{0} \in S_{0}^{P}$,

$$
\operatorname{Ref}\left(\delta_{0}\right)=\left\{\delta_{0}\right\}
$$

$n+1:$ if $\delta=\delta_{0} \wedge \bigwedge_{j \in A g t}\left(\bigwedge_{\delta_{n-1} \in R_{j}\left(\delta_{n}\right)}\left\langle B_{j}\right\rangle \delta_{n} \wedge B_{j} \bigvee_{\delta_{n-1} \in R_{j}\left(\delta_{n}\right)} \delta_{n}\right)$, then

$$
\begin{aligned}
\operatorname{Ref}(\delta)= & \left\{\delta_{0} \wedge \bigwedge_{j \in A g t}\left(\bigwedge_{\delta_{j} \in \operatorname{Ref}\left(R_{j}(\delta)\right)}\left\langle B_{j}\right\rangle \delta_{j} \wedge B_{j} \bigvee_{\delta_{j} \in \operatorname{Ref}\left(R_{j}(\delta)\right)} \delta_{j}\right)\right. \\
& \left.\mid \operatorname{Ref}\left(R_{j}(\delta)\right) \subseteq\left\{\operatorname{Ref}\left(\delta_{j}\right) \mid \delta_{j} \in R_{j}(\delta)\right\}\right\}
\end{aligned}
$$

\footnotetext{
${ }^{3}$ If $R$ is a relation and $n \in \mathbb{N}, R^{\leq n}$ is defined by $R^{\leq n}(w)=\left\{v \mid\right.$ there is $w=w_{0}, \ldots, w_{k}=v$ such that $w_{i} R w_{i+1}$ and $k \leq n\}$ if $n>0$, and $R^{\leq 0}(w)=\{w\}$.
} 


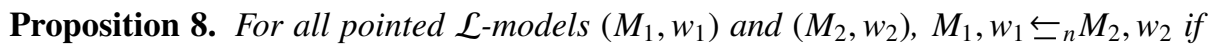
and only if $\delta_{n}\left(w_{2}\right) \in \operatorname{Ref}\left(\delta_{n}\left(w_{1}\right)\right)$.

Proof. By induction on $n$. The case $n=0$ is clear. We prove the induction step. $M_{1}, w_{1} \overleftarrow{-}_{n+1} M_{2}, w_{2}$

iff $\delta_{0}\left(w_{1}\right)=\delta_{0}\left(w_{2}\right)$ and

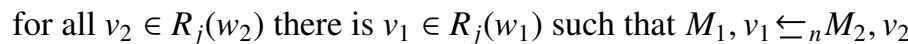

iff $\delta_{0}\left(w_{1}\right)=\delta_{0}\left(w_{2}\right)$ and for all $v_{2} \in R_{j}\left(w_{2}\right)$ there is $v_{1} \in R_{j}\left(w_{1}\right)$ such that $\delta_{n}\left(v_{2}\right) \in \operatorname{Re} f\left(\delta_{n}\left(v_{1}\right)\right)$ by induction hypothesis

iff $\delta_{0}\left(w_{1}\right)=\delta_{0}\left(w_{2}\right)$ and for all $\delta_{n} \in R_{j}\left(\delta_{n+1}\left(w_{2}\right)\right)$ there is $\delta_{n}^{*} \in R_{j}\left(\delta_{n+1}\left(w_{1}\right)\right)$ such that $\delta_{n} \in \operatorname{Re} f\left(\delta_{n}^{*}\right)$ iff $\delta_{0}\left(w_{1}\right)=\delta_{0}\left(w_{2}\right)$ and $R_{j}\left(\delta_{n+1}\left(w_{2}\right)\right) \subseteq\left\{\operatorname{Ref}\left(\delta_{n}\right) \mid \delta_{n} \in R_{j}\left(\delta_{n+1}\left(w_{1}\right)\right)\right\}$

iff $\delta_{n+1}\left(w_{2}\right) \in \operatorname{Ref}\left(\delta_{n+1}\left(w_{1}\right)\right)$.

\section{Definition and axiomatization of $\phi, \phi^{\prime} \models \phi^{\prime \prime}$}

Definition 14 (Inference relation $\phi, \phi^{\prime} \models \phi^{\prime \prime}$ ). Let $\phi, \phi^{\prime \prime} \in \mathcal{L}$ and $\phi^{\prime} \in \mathcal{L}^{\prime}$. The inference relation $\phi, \phi^{\prime}=\phi^{\prime \prime}$ is defined as follows:

$\phi, \phi^{\prime} \models \phi^{\prime \prime} \quad$ iff for all pointed $\mathcal{L}$-model $(M, w)$, and $\mathcal{L}^{\prime}$-model $\left(M^{\prime}, w^{\prime}\right)$ such that $M, w \vDash \operatorname{Pre}\left(w^{\prime}\right)$, if $M, w \vDash \phi$ and $M^{\prime}, w^{\prime} \vDash \phi^{\prime}$ then $(M, w) \otimes\left(M^{\prime}, w^{\prime}\right) \vDash \phi^{\prime \prime}$

We first provide in Section 4.1 a sequent calculus for the case of epistemic events, i.e. events which do not change atomic facts in a situation. The case of ontic events, i.e. events which change atomic facts, will be dealt with in Section 4.2.

\subsection{DEL-Sequent Calculus for epistemic events}

Definition 15 (DEL-Sequent Calculus SC). The DEL-Sequent Calculus SC is defined by the following axiom schemata and inference rules. Below, $\perp$ (resp. T) stands for any $\mathrm{K}$-inconsistent formula (resp. K-theorem), and $\perp^{\prime}$ (resp. $\mathrm{T}^{\prime}$ ) stands for any $\mathrm{K}^{\prime}$ inconsistent formula (resp. $\mathrm{K}^{\prime}$-theorem).

$$
\begin{array}{cccc}
\perp, \phi^{\prime} \vdash \phi^{\prime \prime} & \mathrm{A}_{1} \quad \phi, \perp^{\prime} \vdash \phi^{\prime \prime} & \mathrm{A}_{2} & \phi, \phi^{\prime} \vdash \top \quad \mathrm{A}_{3} \\
p, \top^{\prime} \vdash p & \mathrm{~A}_{4} \quad \neg p, \top^{\prime} \vdash \neg p & \mathrm{~A}_{5} & \neg \operatorname{Pre}\left(p^{\prime}\right), p^{\prime} \vdash \perp \quad \mathrm{A}_{6} \\
\frac{\phi, \phi^{\prime} \vdash \phi^{\prime \prime} \quad \phi, \phi^{\prime} \vdash \phi^{\prime \prime} \rightarrow \psi^{\prime \prime}}{\phi, \phi^{\prime} \vdash \psi^{\prime \prime}} \mathrm{R}_{1} & \frac{\phi \wedge \psi, \phi^{\prime} \vdash \phi^{\prime \prime}}{\phi, \phi^{\prime} \vdash \phi^{\prime} \vdash \phi^{\prime \prime}} \\
\frac{\phi, \phi^{\prime} \wedge \psi^{\prime} \vdash \phi^{\prime \prime} \quad \phi, \neg \psi^{\prime} \vdash \phi^{\prime \prime}}{\phi, \phi^{\prime} \vdash \phi^{\prime \prime}} \mathrm{R}_{3} & \frac{\phi, \phi^{\prime} \vdash \phi^{\prime \prime}}{B_{j} \phi, B_{j} \phi^{\prime} \vdash B_{j} \phi^{\prime \prime}} \mathrm{R}_{4}
\end{array}
$$




$$
\frac{\phi, \phi^{\prime} \vdash \phi^{\prime \prime}}{\left\langle B_{j}\right\rangle\left(\phi \wedge \operatorname{Pre}\left(p^{\prime}\right)\right),\left\langle B_{j}\right\rangle\left(\phi^{\prime} \wedge p^{\prime}\right) \vdash\left\langle B_{j}\right\rangle \phi^{\prime \prime}} \mathrm{R}_{5}
$$

The key axiom schemata and inference rules are $A_{4}, A_{5}, R_{4}$ and $R_{5}$. They permit to build all the valid DEL-sequents by induction on the degree of the formula. Axiom schemata $A_{4}$ and $A_{5}$ can be seen as the base case, and rules $R_{4}$ and $R_{5}$ (together with the rest of the axiom schemata) can be seen as the induction steps allowing to build DEL-sequents of higher degree. Axiom schemata $A_{4}$ and $A_{5}$ also illustrates the fact that we deal as in the standard framework of DEL with epistemic events, i.e. events which do not change atomic facts. We will see in the next section that axiom schemata $A_{4}$ and $A_{5}$ can be adapted in order to deal with ontic events, i.e. events that change atomic facts. Axiom schema $A_{6}$ illustrates the fact that an atomic event can occur only in a possible world where its precondition holds.

These axiom schemata and inference rules provide a formal and accurate analysis of the product update. It turns out that the informal motivations for the definition of the product update in [Baltag \& Moss, 2004] are somehow formalized by rule $R_{5}$. Here is how the product update was informally motivated in this paper (the notations in this quotation are replaced by our notations):

"The update product restricts the full Cartesian product $W \times W^{\prime}$ to the smaller set $W \otimes W^{\prime}$ in order to insure that states survive actions in the appropriate sense. [...] The components of our $\mathcal{L}^{\prime}$-models are "simple actions", so the uncertainty regarding the action is assumed to be independent of the uncertainty regarding the current (input) state. This independence allows us to "multiply" these two uncertainties in order to compute the uncertainty regarding the output state: if whenever the input state is $w$, agent $j$ thinks the input might be some other state $v$, and if whenever the current action happening is $w^{\prime}$, agent $j$ thinks the current action might be some other action $v^{\prime}$, and if $v$ survives $v^{\prime}$, then whenever the output state $\left(w, w^{\prime}\right)$ is reached, agent $j$ thinks the alternative output state $\left(v, v^{\prime}\right)$ might have been reached."

[Baltag \& Moss, 2004]

Now, if one thinks of formulas $\phi, \phi^{\prime}$ and $\phi^{\prime \prime}$ in rule $\mathrm{R}_{5}$ as representing respectively the input state $v$, the action $v^{\prime}$ and the output state $\left(v, v^{\prime}\right)$, then the conclusion of this rule somehow formalizes these informal motivations.

We propose below a second DEL-Sequent Calculus SC*, which will be proved equivalent to the first DEL-Sequent Calculus $S C$ in Section 4.3. Note that Rules $R_{7}$ and $R_{8}$ below are similar to the introduction rules of disjunction of Gentzen's sequent calculus, and rule $R_{6}$ (and in a sense also rules $R_{9}$ and $R_{10}$ ) is similar to the introduction rules of conjunction of Gentzen's sequent calculus.

Definition 16 (DEL-Sequent Calculus SC*). The DEL-Sequent Calculus SC* is defined by the following axiom schemata and inference rules, together with the axiom schemata $A_{2}$ and $A_{6}$ and inference rules $R_{4}$ and $R_{5}$ of the DEL-Sequent Calculus SC. 
Below, $\phi_{p}$ stands for any propositional formula.

$$
\begin{aligned}
& \phi_{p},\left.\top^{\prime}\right|^{*} \phi_{p} \quad \mathrm{~A}_{7} \\
& \frac{\phi, \phi^{\prime} \vdash^{*} \phi^{\prime \prime} \quad \phi, \phi^{\prime} \vdash^{*} \psi^{\prime \prime}}{\phi, \phi^{\prime} \vdash^{*} \phi^{\prime \prime} \wedge \psi^{\prime \prime}} \mathrm{R}_{6} \\
& \frac{\phi, \phi^{\prime} \vdash^{*} \phi^{\prime \prime} \quad \phi,\left.\psi^{\prime}\right|^{*} \phi^{\prime \prime}}{\phi, \phi^{\prime} \vee \psi^{\prime} \vdash^{*} \phi^{\prime \prime}} \mathrm{R}_{7} \\
& \frac{\phi, \phi^{\prime} \vdash^{*} \phi^{\prime \prime} \quad \psi, \phi^{\prime} \vdash^{*} \phi^{\prime \prime}}{\phi \vee \psi, \phi^{\prime} \vdash^{*} \phi^{\prime \prime}} \mathrm{R}_{8} \\
& \frac{\phi, \phi^{\prime} \vdash^{*} \phi^{\prime \prime}}{\psi, \phi^{\prime} \vdash^{*} \psi^{\prime \prime}} \mathrm{R}_{9} \\
& \frac{\phi, \phi^{\prime} \vdash^{*} \phi^{\prime \prime}}{\phi, \psi^{\prime} \vdash^{*} \phi^{\prime \prime}} \mathrm{R}_{10}
\end{aligned}
$$

where $\psi \rightarrow \phi \in \mathrm{K}$ and $\phi^{\prime \prime} \rightarrow \psi^{\prime \prime} \in \mathrm{K}$.

where $\psi^{\prime} \rightarrow \phi^{\prime} \in \mathrm{K}^{\prime}$.

As we said in the introduction, [Baltag et al., 2005, Baltag et al., 2007] provides in an algebraic setting a sequent calculus whose sequents can be arbitrarily long and consist of different types of formulas which can contain propositions, events and agents, and which resolve into a single proposition or event. However, one has to admit that these sequents do not have an easy and intuitive reading. For the specific case of public announcements, a complete axiomatization for a sequent of the form $\phi_{1}, \ldots, \phi_{n} \vdash \psi$ has also been proposed in [van Benthem, 2003]. The reading of this sequent is 'after successive public announcements of the premises, we reach a situation where the announcement of $\psi$ effects no change'. This work is extended in [Cordon-Franco et al., 2010] to take into account a background set of formulas known or commonly known by the agents, which makes it closer to our tripartite approach. However, they do not provide a complete axiomatization of their two consequence relations.

\subsection{DEL-Sequent Calculus for ontic events}

To deal with ontic events, we follow the approach of [van Benthem et al., 2006] and associate to each propositional variable $p^{\prime} \in \Phi^{\prime}$ a substitution function $\operatorname{Sub}\left(p^{\prime}\right): \Phi \rightarrow$ $\mathcal{L}$. Intuitively, $\operatorname{Sub}\left(p^{\prime}\right)(p)$ is a sufficient and necessary condition before the occurrence of $p^{\prime}$ for $p$ to be true after the occurrence of $p^{\prime}$. This substitution function induces a substitution function $\operatorname{Sub}\left(M^{\prime}, w^{\prime}\right)$ over pointed $\mathcal{L}^{\prime}$-models $\left(M^{\prime}, w^{\prime}\right)$ :

$$
\operatorname{Sub}\left(M^{\prime}, w^{\prime}\right)(p)= \begin{cases}\operatorname{Sub}\left(p^{\prime}\right)(p) & \text { if } M^{\prime}, w^{\prime} \vDash p^{\prime} \text { for some } p^{\prime} \in \Phi^{\prime} \\ p & \text { otherwise. }\end{cases}
$$

Then, the new valuation of Equation 5 in Definition 8 is defined as follows:

$$
V^{\otimes}(p)=\left\{\left(v, v^{\prime}\right) \in W^{\otimes} \mid M, v \vDash \operatorname{Sub}\left(M^{\prime}, v^{\prime}\right)(p)\right\} .
$$

One can easily show that this new definition of the product update is axiomatized by replacing axiom schemata $A_{4}$ and $A_{5}$ by the following axiom schemata:

$$
\begin{array}{ll}
\mathrm{A}_{4}^{\prime} & \operatorname{Sub}\left(p^{\prime}\right)(p), p^{\prime} \vdash p \\
\mathrm{~A}_{5}^{\prime} & \neg \operatorname{Sub}\left(p^{\prime}\right)(p), p^{\prime} \vdash \neg p
\end{array}
$$




\subsection{Soundness and completeness of SC and SC*}

Theorem 9 (Soundness of SC). Let $\phi, \phi^{\prime \prime} \in \mathcal{L}$ and $\phi^{\prime} \in \mathcal{L}^{\prime}$. If $\phi, \phi^{\prime} \vdash \phi^{\prime \prime}$ then $\phi, \phi^{\prime} \models \phi^{\prime \prime}$.

Proof. We only prove the soundness of $\mathrm{R}_{4}$ and $\mathrm{R}_{5}$. The proofs of soundness of the other axiom schemata and inference rules are standard.

Soundness of $\mathrm{R}_{4}$. Assume that $\phi, \phi^{\prime} \models \phi^{\prime \prime}$. We are going to prove that $B_{j} \phi, B_{j} \phi^{\prime} \models$ $B_{j} \phi^{\prime \prime}$. Let $(M, w)$ be a pointed $\mathcal{L}$-model and $\left(M^{\prime}, w^{\prime}\right)$ be a pointed $\mathcal{L}^{\prime}$-models such that $M, w \vDash \operatorname{Pre}\left(w^{\prime}\right), M, w \vDash B_{j} \phi$ and $M^{\prime}, w^{\prime} \vDash B_{j} \phi^{\prime}$. Then for all $v \in R_{j}(w) M, v \vDash \phi$ and for all $v^{\prime} \in R_{j}\left(w^{\prime}\right), M^{\prime}, v^{\prime} \vDash \phi^{\prime}$. So for all $v^{\prime} \in R_{j}\left(w^{\prime}\right)$ and all $v \in R_{j}(w)$ such that $M, v \vDash$ $\operatorname{Pre}\left(v^{\prime}\right), M, v \vDash \phi$ and $M^{\prime}, v^{\prime} \vDash \phi^{\prime}$. So for all $\left(v, v^{\prime}\right) \in R_{j}\left(w, w^{\prime}\right),(M, v) \otimes\left(M^{\prime}, v^{\prime}\right) \vDash \phi^{\prime \prime}$ by definition of $\phi, \phi^{\prime} \models \phi^{\prime \prime}$. So $(M, w) \otimes\left(M^{\prime}, w^{\prime}\right) \models B_{j} \phi^{\prime \prime}$. Therefore $B_{j} \phi, B_{j} \phi^{\prime} \models B_{j} \phi^{\prime \prime}$.

Soundness of $\mathrm{R}_{5}$. Assume that $\phi, \phi^{\prime} \models \phi^{\prime \prime}$. We are going to prove that $\left\langle B_{j}\right\rangle(\phi \wedge$ $\left.\operatorname{Pr}\left(p^{\prime}\right)\right),\left\langle B_{j}\right\rangle\left(\phi^{\prime} \wedge p^{\prime}\right) \models\left\langle B_{j}\right\rangle \phi^{\prime \prime}$. Let $(M, w)$ be a pointed $\mathcal{L}$-model and $\left(M^{\prime}, w^{\prime}\right)$ be a pointed $\mathcal{L}^{\prime}$-model such that $M, w \vDash \operatorname{Pre}\left(w^{\prime}\right), M, w \vDash\left\langle B_{j}\right\rangle\left(\phi \wedge \operatorname{Pre}\left(p^{\prime}\right)\right)$ and $M^{\prime}, w^{\prime} \vDash$ $\left\langle B_{j}\right\rangle\left(\phi^{\prime} \wedge p^{\prime}\right)$. Then there is $v^{\prime} \in R_{j}\left(w^{\prime}\right)$ such that $M^{\prime}, v^{\prime} \vDash \phi^{\prime}$ and there is $v \in R_{j}(w)$ such that $M, v \vDash \phi \wedge \operatorname{Pre}\left(v^{\prime}\right)$. Therefore $(M, v) \otimes\left(M^{\prime}, v^{\prime}\right) \models \phi^{\prime \prime}$ because $\phi, \phi^{\prime} \models \phi^{\prime \prime}$. So $(M, w) \otimes\left(M^{\prime}, w^{\prime}\right) \vDash\left\langle B_{j}\right\rangle \phi^{\prime \prime}$ because $\left(v, v^{\prime}\right) \in R_{j}\left(w, w^{\prime}\right)$.

The proof of completeness of Theorem 14 is based on the following rough ideas. For any $\phi, \phi^{\prime \prime} \in \mathcal{L}$ and $\phi^{\prime} \in \mathcal{L}^{\prime}$, the DEL-sequent $\phi, \phi^{\prime} \vdash \phi^{\prime \prime}$ is provably equivalent to a disjunction of $\delta, \delta^{\prime} \vdash \delta^{\prime \prime}$, because formulas $\phi, \phi^{\prime}$ and $\phi^{\prime \prime}$ are themselves provably equivalent to a disjunction of $\delta \mathrm{s}, \delta^{\prime} \mathrm{s}$ and $\delta^{\prime \prime}$ s respectively. Hence, if we prove completeness for these normal form formulas, we will inherit the completeness for the full epistemic and event languages by soundness of SC. Proposition 13 proves this completeness result for normal form formulas. It relies on Lemmas 10 and 11 which define a set of axiom schemata and rules derivable in SC, and which extend those of SC*. Even if these axiom schemata and inference rules are less intuitive than those of SC, they will turn out to be more appropriate to prove the completeness of SC.

Lemma 10. For all $\phi, \phi^{\prime \prime} \in \mathcal{L}$ and all $\phi^{\prime} \in \mathcal{L}^{\prime}, \phi,\left.\phi^{\prime}\right|^{*} \phi^{\prime \prime}$ implies $\phi, \phi^{\prime} \vdash \phi^{\prime \prime}$.

Proof (Sketch). It suffices to prove that axiom $A_{7}$ and rules $R_{6}, R_{7}, R_{8}, R_{9}$ and $R_{10}$ from the DEL-Sequent Calculus SC* are all derivable in SC. Rule $R_{10}$ is derivable from $A_{2}$ and $R_{3}$ by considering $\perp=\psi^{\prime} \wedge \neg \phi^{\prime}$. The first (resp. second) part of $R_{9}$ is derivable from $A_{1}$ (resp. $A_{3}$ ) and $R_{1}$ (resp. $R_{2}$ ) by considering $\perp=\psi \wedge \neg \phi$ (resp. $\perp=\psi^{\prime \prime} \wedge \neg \phi^{\prime \prime}$ ). Rule $R_{6}$ is derivable from $A_{3}$ and $R_{1}$ by considering $T=\phi^{\prime \prime} \rightarrow\left(\psi^{\prime \prime} \rightarrow\left(\phi^{\prime \prime} \wedge \psi^{\prime \prime}\right)\right)$. Rule $R_{7}$ is derivable from $A_{2}$ and $R_{3}$ by considering $\perp^{\prime}=\left(\phi^{\prime} \vee \psi^{\prime}\right) \wedge \neg \phi^{\prime} \wedge \neg \psi^{\prime}$. Rule $R_{8}$ is derivable from $A_{1}$ and $R_{2}$ by considering $\perp=(\phi \vee \psi) \wedge \neg \phi \wedge \neg \psi$.

Lemma 10 entails that in order to prove completeness of SC, we can equivalently use the axiom schemata and inference rules of SC*. In the same line, Lemma 11 below entails that to prove completeness of $\mathrm{SC}$, we can equivalently use the inference rule $\mathrm{R}_{10}$ or $\mathrm{R}_{11}$.

Lemma 11. Let $P^{\prime}$ be a finite subset of $\Phi^{\prime}$. Let $\phi, \phi^{\prime \prime} \in \mathcal{L}$ and $\phi^{\prime}, \psi^{\prime} \in \mathcal{L}^{\prime}$ be such that $P^{\prime}\left(\phi^{\prime}\right) \cup P^{\prime}\left(\psi^{\prime}\right) \subset P^{\prime}$. If $\psi^{\prime} \rightarrow \phi^{\prime} \in K^{P^{\prime}}$, then one can prove the following derivation in 
SC:

$$
\frac{\phi, \phi^{\prime} \vdash \phi^{\prime \prime}}{\phi, \psi^{\prime} \vdash \phi^{\prime \prime}} R_{11}
$$

Proof. It follows from Proposition 6 that $\psi^{\prime} \rightarrow \phi^{\prime} \in \mathrm{K}^{\mathrm{P}^{\prime}}$ if and only if $\psi^{\prime} \rightarrow \phi^{\prime} \in \mathrm{K}^{\prime}$, because $P^{\prime}\left(\psi^{\prime} \rightarrow \phi^{\prime}\right)=P^{\prime}\left(\psi^{\prime}\right) \cup P^{\prime}\left(\phi^{\prime}\right) \subset P^{\prime}$. We then immediately get rule $\mathrm{R}_{11}$ by application of rule $\mathrm{R}_{10}$.

Lemma 12. Let $P^{\prime}$ be a finite subset of $\Phi^{\prime}$. Let $\delta_{n}^{\prime} \in E_{n}^{P^{\prime}}$ and let $\phi \in \mathcal{L}$ be such that $\operatorname{deg}(\phi) \leq n$. Let $P=P(\phi) \cup \bigcup\left\{P\left(\operatorname{Pre}\left(p^{\prime}\right)\right) \mid p^{\prime} \in P^{\prime}\right\}$ and let $N=\max \left\{\operatorname{deg}\left(\operatorname{Pre}\left(p^{\prime}\right)\right) \mid p^{\prime} \in P^{\prime}\right\}$ and $\delta_{n+N} \in S_{n+N}^{P}$. Then, $\delta_{n+N}, \delta_{n}^{\prime} \models \phi$ does not hold if and only if $\delta_{n+N}, \delta_{n}^{\prime} \models \neg \phi$ and $\delta_{n+N} \rightarrow \operatorname{Pre}\left(\delta_{n}^{\prime}\right) \in K$.

Proof (Sketch). This lemma can be rephrased as follows: there are a $\mathcal{L}$-model $(M, w)$ and a $\mathcal{L}^{\prime}$-model $\left(M^{\prime}, w^{\prime}\right)$ such that $M, w \vDash \operatorname{Pre}\left(w^{\prime}\right), M, w \vDash \delta_{n+N}, M^{\prime}, w^{\prime} \vDash \delta_{n}^{\prime}$ and $(M, w) \otimes\left(M^{\prime}, w^{\prime}\right) \vDash \phi$ if and only if $\delta_{n+N} \rightarrow \operatorname{Pre}\left(\delta_{n}^{\prime}\right) \in \mathrm{K}$ and for all $\mathcal{L}$-model $(M, w)$ and $\mathcal{L}^{\prime}$-model $\left(M^{\prime}, w^{\prime}\right)$ such that $M, w \vDash \operatorname{Pre}\left(w^{\prime}\right), M, w \vDash \delta_{n+N}$ and $M^{\prime}, w^{\prime} \vDash \delta_{n}^{\prime}$, we have that $(M, w) \otimes\left(M^{\prime}, w^{\prime}\right) \vDash \phi$.

The proof then follows from the fact that the structure of $(M, w) \otimes\left(M^{\prime}, w^{\prime}\right)$ up to modal depth $n$ and for the atomic facts $P(\phi)$ is determined only by the structure of $(M, w)$ up to modal depth $n+N$ and for the atomic facts $P$ and the modal structure of $\left(M^{\prime}, w^{\prime}\right)$ up to modal depth $n$ and for the atomic events $P^{\prime}$, which are themselves completely determined by some $\delta_{n+N} \in S_{n+N}^{P}$ and $\delta_{n}^{\prime} \in E_{n}^{P^{\prime}}$ respectively.

The crucial Proposition 13 proves the soundness and completeness of SC for normal form formulas. The proof of this proposition is by induction on the degrees of the formulas $\delta, \delta^{\prime}$ and $\delta^{\prime \prime}$. The base case is dealt with by Axiom $\mathrm{A}_{7}$. The induction step is proved by resorting to the crucial inference rules $R_{4}$ and $R_{5}$, and Lemma 12 .

Proposition 13. Let $P^{\prime}$ be a finite subset of $\Phi^{\prime}$ and let $P$ be a finite subset of $\Phi$ such that $\bigcup\left\{P\left(\operatorname{Pre}\left(p^{\prime}\right)\right) \mid p^{\prime} \in P^{\prime}\right\} \subseteq P$. Let $N=\max \left\{\operatorname{deg}\left(\operatorname{Pre}\left(p^{\prime}\right)\right) \mid p^{\prime} \in P^{\prime}\right\}$. For all $\delta_{n+N} \in S_{n+N}^{P}, \delta_{n}^{\prime} \in E_{n}^{P^{\prime}}$ and $\delta_{n}^{\prime \prime} \in S_{n}^{P}$ such that $\delta_{n+N} \rightarrow \operatorname{Pre}\left(\delta_{n}^{\prime}\right) \in K$, it holds that $\delta_{n+N}, \delta_{n}^{\prime} \vdash \delta_{n}^{\prime \prime}$ if and only if $\delta_{n+N}, \delta_{n}^{\prime} \models \delta_{n}^{\prime \prime}$.

Proof. We prove it by induction on $n$. The left to right direction holds by soundness of Theorem 9 . We only prove the right to left direction.

$\mathbf{n}=\mathbf{0}$. Assume that $\delta_{N}, \delta_{0}^{\prime} \models \delta_{0}^{\prime \prime}$. Then, because $\delta_{N} \rightarrow \operatorname{Pre}\left(\delta_{0}^{\prime}\right) \in \mathrm{K}$, there do exist a pointed $\mathcal{L}$-model $(M, w)$ and a pointed $\mathcal{L}^{\prime}$-model $\left(M^{\prime}, w^{\prime}\right)$ such that $M, w \vDash \delta_{N}$, $M, w \vDash \operatorname{Pre}\left(w^{\prime}\right)$ and $M^{\prime}, w^{\prime} \vDash \delta_{0}^{\prime}$. Therefore, because the product update of Definition 8 does not change the valuations, $\delta_{N} \rightarrow \delta_{0}^{\prime \prime} \in \mathrm{K}$. Now, $\delta_{0}^{\prime \prime}, \delta_{0}^{\prime} \vdash \delta_{0}^{\prime \prime}$ by Axiom $\mathrm{A}_{7}$. So, by application of rule $\mathrm{R}_{9}$ and because $\delta_{N} \rightarrow \delta_{0}^{\prime \prime} \in \mathrm{K}$, it holds that $\delta_{N}, \delta_{0}^{\prime} \vdash \delta_{0}^{\prime \prime}$. 
$\mathbf{n}+$ 1. Let $\delta_{n+1+N} \in S_{n+1+N}^{P}, \delta_{n+1}^{\prime} \in E_{n+1}^{P^{\prime}}$ and $\delta_{n+1}^{\prime \prime} \in S_{n+1}^{P}$ such that $\delta_{n+1+N} \rightarrow \operatorname{Pre}\left(\delta_{n+1}^{\prime}\right) \in$ $\mathrm{K}$ :

$$
\begin{aligned}
\delta_{n+1+N} & =\delta_{0} \wedge \bigwedge_{j \in A g t}\left(\bigwedge_{i \in I}\left\langle B_{j}\right\rangle \delta_{n+N, i} \wedge B_{j}\left(\bigvee_{i \in I} \delta_{n+N, i}\right)\right) \\
\delta_{n+1}^{\prime} & =\delta_{0}^{\prime} \wedge \bigwedge_{j \in A g t}\left(\bigwedge_{k \in I^{\prime}}\left\langle B_{j}\right\rangle \delta_{n, k}^{\prime} \wedge B_{j}\left(\bigvee_{k \in I^{\prime}} \delta_{n, k}^{\prime}\right)\right) \\
\delta_{n+1}^{\prime \prime} & =\delta_{0}^{\prime \prime} \wedge \bigwedge_{j \in A g t}\left(\bigwedge_{i \in I^{\prime \prime}}\left\langle B_{j}\right\rangle \delta_{n, i}^{\prime \prime} \wedge B_{j}\left(\bigvee_{i \in I^{\prime \prime}} \delta_{n, i}^{\prime \prime}\right)\right) .
\end{aligned}
$$

By assumption, $\delta_{n+1+N}, \delta_{n+1}^{\prime} \models \delta_{n+1}^{\prime \prime}$. Assume towards a contradiction that $\delta_{n+1+N}, \delta_{n+1}^{\prime} \vdash \delta_{n+1}^{\prime \prime}$ does not hold. Then, because of rule $\mathrm{R}_{6}$ :

$$
\begin{aligned}
& \text { either } \delta_{n+1+N}, \delta_{n+1}^{\prime} \vdash \delta_{0}^{\prime \prime} \text { does not hold, } \\
& \text { or } \delta_{n+1+N}, \delta_{n+1}^{\prime} \vdash\left\langle B_{j}\right\rangle \delta_{n, i}^{\prime \prime} \text { does not hold for some } i \in I^{\prime \prime} \\
& \text { or } \delta_{n+1+N}, \delta_{n+1}^{\prime} \vdash B_{j}\left(\bigvee_{i \in I^{\prime \prime}} \delta_{n, i}^{\prime \prime}\right) \text { does not hold }
\end{aligned}
$$$$
\text { (Case 1) }
$$

We are going to show that these three cases are all impossible.

(Case 3): Assume that $\delta_{n+1+N}, \delta_{n+1}^{\prime} \vdash B_{j}\left(\bigvee_{i \in I^{\prime \prime}} \delta_{n, i}^{\prime \prime}\right)$ does not hold. Then, by application of rules $\mathrm{R}_{9}$ and $\mathrm{R}_{10}$, because $\delta_{n+1+N} \rightarrow B_{j}\left(\bigvee_{i \in I} \delta_{n+N, i}\right) \in \mathrm{K}$ and $\delta_{n+1}^{\prime} \rightarrow$ $B_{j}\left(\bigvee_{k \in I^{\prime}} \delta_{n, k}^{\prime}\right) \in \mathrm{K}^{\prime}$, we have that $B_{j}\left(\bigvee_{i \in I} \delta_{n+N, i}\right), B_{j}\left(\bigvee_{k \in I^{\prime}} \delta_{n, k}^{\prime}\right) \vdash B_{j}\left(\bigvee_{i \in I^{\prime \prime}} \delta_{n, i}^{\prime \prime}\right)$ does not hold. Then, by application of rule $R_{4}$,

$$
\bigvee_{i \in I} \delta_{n+N, i}, \bigvee_{k \in I^{\prime}} \delta_{n, k}^{\prime} \vdash \bigvee_{i \in I^{\prime \prime}} \delta_{n, i}^{\prime \prime} \text { does not hold }
$$

We now show that $\delta_{n+N, i_{0}}, \delta_{n, k_{0}}^{\prime} \vdash \bigvee_{i \in I^{\prime \prime}} \delta_{n, i}^{\prime \prime}$ does not hold for some $\delta_{n+N, i_{0}}$ and $\delta_{n, k_{0}}^{\prime}$ such that $\delta_{n+N, i_{0}} \rightarrow \operatorname{Pre}\left(\delta_{n, k_{0}}^{\prime}\right) \in \mathrm{K}$. Assume towards a contradiction that for all $\delta_{n+N, i}$ and $\delta_{n, k}^{\prime}$ such that $\delta_{n+N, i} \rightarrow \operatorname{Pre}\left(\delta_{n, k}^{\prime}\right) \in \mathrm{K}$, we have that $\delta_{n+N, i}, \delta_{n, k}^{\prime} \vdash \bigvee_{i \in I^{\prime \prime}} \delta_{n, i}^{\prime \prime}$. Let $\delta_{n+N, i}$ and $\delta_{n, i}$ be such that $\delta_{n+N, i} \rightarrow \operatorname{Pre}\left(\delta_{n, k}^{\prime}\right) \notin \mathrm{K}$. We are going to show that $\delta_{n+N, i}, \delta_{n, k}^{\prime} \vdash \bigvee_{i \in I^{\prime \prime}} \delta_{n, i}^{\prime \prime}$ holds as well in this case. First, by Proposition 3 , $\delta_{n+N, i} \rightarrow \neg \operatorname{Pre}\left(\delta_{n, k}^{\prime}\right) \in \mathrm{K}$. Now, by definition of $E_{n}^{P^{\prime}}$, there is $p^{\prime} \in P^{\prime}$ such that $\delta_{n, k}^{\prime} \rightarrow p^{\prime} \in \mathrm{K}^{\prime}$. By Axiom $\mathrm{A}_{6}, \neg \operatorname{Pre}\left(p^{\prime}\right), p^{\prime} \vdash \perp$. Therefore, $\neg \operatorname{Pre}\left(\delta_{n, k}^{\prime}\right), p^{\prime} \vdash \perp$ because $\operatorname{Pre}\left(\delta_{n, k}^{\prime}\right)=\operatorname{Pre}\left(p^{\prime}\right)$. Then, by application of Rules $\mathrm{R}_{9}$ and $\mathrm{R}_{10}$, and 
because $\delta_{n, k}^{\prime} \rightarrow p^{\prime} \in \mathrm{K}^{\prime}, \delta_{n+N, i} \rightarrow \neg \operatorname{Pre}\left(\delta_{n, k}^{\prime}\right) \in \mathrm{K}$ and $\perp \rightarrow \bigvee_{i \in I^{\prime \prime}} \delta_{n, i}^{\prime \prime} \in \mathrm{K}$, we obtain $\delta_{n+N, i}, \delta_{n, k}^{\prime} \vdash \bigvee_{i \in I^{\prime \prime}} \delta_{n, i}^{\prime \prime}$. Hence, for all $\delta_{n+N, i}$ and $\delta_{n, k}^{\prime}$, we have that $\delta_{n+N, i}, \delta_{n, k}^{\prime} \vdash \bigvee_{i \in I^{\prime \prime}} \delta_{n, i}^{\prime \prime}$. Therefore, by application of rules $\mathrm{R}_{7}$ and $\mathrm{R}_{8}$, we have that $\bigvee_{i \in I} \delta_{n+N, i}, \bigvee_{k \in I^{\prime}} \delta_{n, k}^{\prime}{ }^{i \in I^{\prime \prime}} \bigvee_{i \in I^{\prime \prime}} \delta_{n, i}^{\prime \prime}$.

This is impossible because of Equation 15. Therefore, $\delta_{n+N, i_{0}}, \delta_{n, k_{0}}^{\prime} \vdash \bigvee_{i \in I^{\prime \prime}} \delta_{n, i}^{\prime \prime}$ does not hold for some $\delta_{n+N, i_{0}}$ and $\delta_{n, k_{0}}^{\prime}$ such that $\delta_{n+N, i_{0}} \rightarrow \operatorname{Pre}\left(\delta_{n, k_{0}}^{\prime}\right) \in \mathrm{K}$.

So for all $i \in I^{\prime \prime}, \delta_{n+N, i_{0}}, \delta_{n, k_{0}}^{\prime} \vdash \delta_{n, i}^{\prime \prime}$ does not hold by application of rule $\mathrm{R}_{9}$. Then, for all $i \in I^{\prime \prime}, \delta_{n+N, i_{0}}, \delta_{n, k_{0}}^{\prime} \models \delta_{n, i}^{\prime \prime}$ does not hold by induction hypothesis. Then, by Lemma 12 , for all $i \in I^{\prime \prime}, \delta_{n+N, i_{0}}, \delta_{n, k_{0}}^{\prime} \models \neg \delta_{n, i}^{\prime \prime}$. So $\delta_{n+N, i_{0}}, \delta_{n, k_{0}}^{\prime} \models \bigwedge_{i \in I^{\prime \prime}} \neg \delta_{n, i}^{\prime \prime}$. So, $\left\langle B_{j}\right\rangle \delta_{n+N, i_{0}},\left\langle B_{j}\right\rangle \delta_{n, k_{0}}^{\prime} \models\left\langle B_{j}\right\rangle \bigwedge_{i \in I^{\prime \prime}} \neg \delta_{n, i}^{\prime \prime}$ by soundness of rule $R_{5}$, because $\delta_{n+N, i_{0}} \rightarrow$ $\operatorname{Pre}\left(\delta_{n, k_{0}}^{\prime}\right) \in \mathrm{K}$ by assumption. Therefore, $\left\langle B_{j}\right\rangle \delta_{n+N, i_{0}},\left\langle B_{j}\right\rangle \delta_{n, k_{0}}^{\prime} \models \neg B_{j}\left(\bigvee_{i \in I^{\prime \prime}} \delta_{n, i}^{\prime \prime}\right)$. Then $\delta_{n+1+N}, \delta_{n+1}^{\prime} \models \neg \delta_{n+1}^{\prime \prime}$ by soundness of Rules $\mathrm{R}_{9}$ and $\mathrm{R}_{10}$ and because $\delta_{n+1+N} \rightarrow$ $\left\langle B_{j}\right\rangle \delta_{n+N, i_{0}} \in \mathrm{K}, \delta_{n+1}^{\prime} \rightarrow\left\langle B_{j}\right\rangle \delta_{n, k_{0}}^{\prime} \in \mathrm{K}^{\prime}$ and $\neg B_{j}\left(\bigvee_{i \in I^{\prime \prime}} \delta_{n, i}^{\prime \prime}\right) \rightarrow \neg \delta_{n+1}^{\prime \prime} \in \mathrm{K}$. Now, by assumption, we have that $\delta_{n+1+N} \rightarrow \operatorname{Pre}\left(\delta_{n+1}^{\prime}\right) \in \mathrm{K}$. Therefore, by Lemma 12, we have that $\delta_{n+1+N}, \delta_{n+1}^{\prime} \models \delta_{n+1}^{\prime \prime}$ does not hold. This is impossible by assumption. Therefore, (Case 3) cannot hold.

(Case 2): Assume that $\delta_{n+1+N}, \delta_{n+1}^{\prime} \vdash\left\langle B_{j}\right\rangle \delta_{n, l}^{\prime \prime}$ does not hold for some $l \in I^{\prime \prime}$. By application of $\mathrm{R}_{9}$, because $\delta_{n+1+N} \rightarrow\left\langle B_{j}\right\rangle \delta_{n+N, i} \in \mathrm{K}$ and $\delta_{n+1}^{\prime} \rightarrow\left\langle B_{j}\right\rangle \delta_{n, k}^{\prime} \in \mathrm{K}^{\prime}$, we obtain that $\left\langle B_{j}\right\rangle \delta_{n+N, i},\left\langle B_{j}\right\rangle \delta_{n, k}^{\prime} \models\left\langle B_{j}\right\rangle \delta_{n, l}^{\prime \prime}$ does not hold for all $i \in I$ and $k \in I^{\prime}$. We are going to show that $\delta_{n+N, i}, \delta_{n, k}^{\prime} \models \neg \delta_{n, l}^{\prime \prime}$ for all $i \in I$ and $k \in I^{\prime}$.

1. Let $i \in I$ and $k \in I^{\prime}$ and assume that $\delta_{n+N, i} \rightarrow \neg \operatorname{Pre}\left(\delta_{n, k}^{\prime}\right) \in \mathrm{K}$. Let $\delta_{0}^{\prime}$ be the propositional part of $\delta_{n, k}^{\prime}$. By $\mathrm{A}_{6}$, we have that $\neg \operatorname{Pre}\left(\delta_{0}^{\prime}\right), \delta_{0}^{\prime} \vdash \perp$. Then, because $\operatorname{Pre}\left(\delta_{0}^{\prime}\right)=\operatorname{Pre}\left(\delta_{n, k}^{\prime}\right)$, we obtain $\neg \operatorname{Pre}\left(\delta_{n, k}^{\prime}\right), \delta_{0}^{\prime} \vdash \perp$. Then, by application of $\mathrm{R}_{10}$, because $\delta_{n, k}^{\prime} \rightarrow \delta_{0}^{\prime} \in \mathrm{K}^{\prime}$, we obtain $\neg \operatorname{Pre}\left(\delta_{n, k}^{\prime}\right), \delta_{n, k}^{\prime} \vdash \perp$. Therefore, by application of $\mathrm{R}_{9}$, because $\delta_{n+N, i} \rightarrow \neg \operatorname{Pre}\left(\delta_{n, k}^{\prime}\right) \in \mathrm{K}$ and $\perp \rightarrow \neg \delta_{n, l}^{\prime \prime} \in \mathrm{K}$, we obtain $\delta_{n+N, i}, \delta_{n, k} \vdash \neg \delta_{n, l}^{\prime \prime}$. Finally, by soundness of SC, $\delta_{n+N, i}, \delta_{n, k} \models \neg \delta_{n, l}^{\prime \prime}$.

2. Let $i \in I$ and $k \in I^{\prime}$ and assume that $\delta_{n+N, i} \rightarrow \operatorname{Pre}\left(\delta_{n, k}^{\prime}\right) \in \mathrm{K}$. Then $\left\langle B_{j}\right\rangle \delta_{n+N, i} \rightarrow\left\langle B_{j}\right\rangle\left(\delta_{n+N, i} \wedge \operatorname{Pre}\left(\delta_{n, k}^{\prime}\right)\right) \in \mathrm{K}$. Because $\left\langle B_{j}\right\rangle \delta_{n+N, i},\left\langle B_{j}\right\rangle \delta_{n, k}^{\prime} \models\left\langle B_{j}\right\rangle \delta_{n, l}^{\prime \prime}$ does not hold, we have that $\left\langle B_{j}\right\rangle\left(\delta_{n+N, i} \wedge \operatorname{Pre}\left(\delta_{n, k}^{\prime}\right)\right),\left\langle B_{j}\right\rangle \delta_{n, k}^{\prime} \vdash\left\langle B_{j}\right\rangle \delta_{n, l}^{\prime \prime}$ does not hold either, by application of rule $\mathrm{R}_{9}$. Then $\delta_{n+N, i}, \delta_{n, k}^{\prime} \vdash \delta_{n, l}^{\prime \prime}$ does not hold, by application of rule $\mathrm{R}_{5}$. So $\delta_{n+N, i}, \delta_{n, k}^{\prime} \models \delta_{n, l}^{\prime \prime}$ does not hold by Induction Hypothesis. Then, by Lemma $12, \delta_{n+N, i}, \delta_{n, k}^{\prime} \models \neg \delta_{n, l}^{\prime \prime}$. 
Consequently, by application of rules $\mathrm{R}_{8}$ and $\mathrm{R}_{7}$, for some $l \in I^{\prime \prime} \bigvee_{i \in I} \delta_{n+N, i}, \bigvee_{k \in I^{\prime}} \delta_{n, k}^{\prime} \models \neg \delta_{n, l}^{\prime \prime}$. Then, $B_{j}\left(\bigvee_{i \in I} \delta_{n+N, i}\right), B_{j}\left(\bigvee_{k \in I^{\prime}} \delta_{n, k}^{\prime}\right) \models \neg\left\langle B_{j}\right\rangle \delta_{n, l}^{\prime \prime}$ by rule $\mathrm{R}_{4}$. So $\delta_{n+N+1}, \delta_{n+1}^{\prime} \models \neg \delta_{n+1}^{\prime \prime}$ by rule $\mathrm{R}_{9}$, because $\delta_{n+N+1} \rightarrow B_{j}\left(\bigvee_{i \in I} \delta_{n+N, i}\right) \in \mathrm{K}, \delta_{n+1}^{\prime} \rightarrow B_{j}\left(\bigvee_{k \in I^{\prime}} \delta_{n, k}^{\prime}\right) \in \mathrm{K}^{\prime}$ and $\neg\left\langle B_{j}\right\rangle \delta_{n, l}^{\prime \prime} \rightarrow \delta_{n+1}^{\prime \prime} \in \mathrm{K}$. Therefore, $\delta_{n+N+1}, \delta_{n+1}^{\prime} \models \delta_{n+1}^{\prime \prime}$ does not hold by application of Lemma 12, because by assumption $\delta_{n+N+1} \rightarrow \operatorname{Pre}\left(\delta_{n+1}^{\prime}\right) \in \mathrm{K}$. Consequently, (Case 2) cannot hold.

(Case 1): Assume that $\delta_{n+1+N}, \delta_{n+1}^{\prime} \vdash \delta_{0}^{\prime \prime}$ does not hold. Assume towards a contradiction that $\delta_{n+1+N} \rightarrow \delta_{0}^{\prime \prime} \in \mathrm{K}$. Then, because $\delta_{0}^{\prime \prime}, \delta_{n+1}^{\prime} \vdash \delta_{0}^{\prime \prime}$ holds by axiom $\mathrm{A}_{7}$, we have $\delta_{n+1+N}, \delta_{n+1}^{\prime} \vdash \delta_{0}^{\prime \prime}$ by rule $\mathrm{R}_{9}$, which is impossible. Therefore, $\delta_{n+1+N} \rightarrow \delta_{0}^{\prime \prime} \notin \mathrm{K}$, and so $\delta_{n+1+N} \rightarrow \neg \delta_{0}^{\prime \prime} \in \mathrm{K}$ by Proposition 3. Then, because $\neg \delta_{0}^{\prime \prime}, \delta_{n+1}^{\prime} \models \neg \delta_{0}^{\prime \prime}$ by axiom $\mathrm{A}_{7}, \delta_{n+1+N}, \delta_{n+1}^{\prime} \models \neg \delta_{0}$ by rule $\mathrm{R}_{9}$. So, by application of Lemma 12 and because $\delta_{n+1+N} \rightarrow \operatorname{Pre}\left(\delta_{n+1}^{\prime}\right) \in \mathrm{K}$ by assumption, we obtain that $\delta_{n+1+N}, \delta_{n+1}^{\prime} \models \delta_{0}^{\prime \prime}$ does not hold, which is impossible. Therefore, (Case 1) cannot hold.

Because all three cases are impossible, it is also impossible that $\delta_{n+1+N}, \delta_{n+1}^{\prime} \vdash \delta_{n+1}^{\prime \prime}$ does not hold. Therefore, $\delta_{n+1+N}, \delta_{n+1}^{\prime} \vdash \delta_{n+1}^{\prime \prime}$ holds.

We have just proved the completeness of SC for normal form formulas. We are going to lift this completeness result of SC to the full epistemic and event languages relying on the fact that any DEL-sequent $\phi, \phi^{\prime} \vdash \phi^{\prime \prime}$ can be reduced equivalently to a disjunction of $\delta, \delta^{\prime} \vdash \delta^{\prime \prime}$, and using the soundness of rules $\mathrm{R}_{7}, \mathrm{R}_{8}, \mathrm{R}_{9}$ and $\mathrm{R}_{11}$.

Theorem 14 (Completeness of SC). Let $\phi, \phi^{\prime \prime} \in \mathcal{L}$ and $\phi^{\prime} \in \mathcal{L}^{\prime}$. If $\phi, \phi^{\prime} \models \phi^{\prime \prime}$ then $\phi, \phi^{\prime} \vdash \phi^{\prime \prime}$.

Proof. Let $\phi, \phi^{\prime \prime} \in \mathcal{L}$, and $\phi^{\prime} \in \mathcal{L}^{\prime}$ such that $\max \left\{\operatorname{deg}(\phi), \operatorname{deg}\left(\phi^{\prime}\right), \operatorname{deg}\left(\phi^{\prime \prime}\right)\right\} \leq n$. Assume that $\phi, \phi^{\prime} \models \phi^{\prime \prime}$.

1. Assume that $\phi^{\prime}$ is $K^{\prime}$-inconsistent. Then, by axiom schemata $A_{2}, \phi, \phi^{\prime} \vdash \phi^{\prime \prime}$.

2. Assume that $\phi^{\prime}$ is $K^{\prime}$-consistent. Let $P^{\prime}$ be a finite subset of $\Phi^{\prime}$ such that $P^{\prime}\left(\phi^{\prime}\right) \subset$ $P^{\prime}$. Then, by Proposition 6, $\phi^{\prime}$ is $K^{P^{\prime}}$-consistent. Let $P=P(\phi) \cup P\left(\phi^{\prime \prime}\right) \cup$ $\bigcup\left\{P\left(\operatorname{Pre}\left(p^{\prime}\right)\right) \mid p^{\prime} \in P^{\prime}\right\}$. Therefore, by Corollaries 1 and 3 , there are $\delta_{n+N, i} \in$ $S_{n}^{P}, \delta_{n, k}^{\prime} \in E_{n}^{P^{\prime}}$ and $\delta_{n, l}^{\prime \prime} \in S_{n}^{P}$ such that $\phi \leftrightarrow \bigvee_{i \in I} \delta_{n+N, i} \in K, \phi^{\prime} \leftrightarrow \bigvee_{k \in I^{\prime}} \delta_{n, k}^{\prime} \in K^{P^{\prime}}$ and $\phi^{\prime \prime} \leftrightarrow \bigvee_{l \in I^{\prime \prime}} \delta_{n, l}^{\prime \prime} \in K$. Then, $\phi^{\prime} \leftrightarrow \bigvee_{k \in I^{\prime}} \delta_{n, k}^{\prime} \in K^{P^{\prime \prime}}$ for any $P^{\prime} \subset P^{\prime \prime}$. Therefore, by soundness of rule $R_{9}$ and rule $R_{11}$ of Lemma 11, $\phi, \phi^{\prime} \models \phi^{\prime \prime}$ iff $\bigvee_{i \in I} \delta_{n+N, i}, \bigvee_{k \in I^{\prime}} \delta_{n, k}^{\prime} \models \bigvee_{l \in I^{\prime \prime}} \delta_{n, l}^{\prime \prime}$.

Then, still by soundness of rules $R_{7}, R_{8}, R_{9}$ and $R_{11}$, we have that $\phi, \phi^{\prime} \models \phi^{\prime \prime}$ iff $\delta_{n+N, i}, \delta_{n, k}^{\prime} \models \bigvee_{l \in I^{\prime \prime}} \delta_{n, l}^{\prime \prime}$ for all $i \in I$ and $k \in I^{\prime}$. Therefore, for all $i \in I$ and $k \in I^{\prime}$, 


$$
\begin{aligned}
& \delta_{n+N, i}, \delta_{n, k}^{\prime} \models \bigvee_{l \in I^{\prime \prime}} \delta_{n, l}^{\prime \prime}(*) \text {. Now, we are going to show that for all } i \in I \text { and } k \in I^{\prime}, \\
& \delta_{n+N, i}, \delta_{n, k}^{\prime} \vdash \bigvee_{l \in I^{\prime \prime}} \delta_{n, l}^{\prime \prime} \text {. Let } i \in I \text { and } k \in I^{\prime} .
\end{aligned}
$$

(a) First, assume that $\delta_{n+N, i} \rightarrow \operatorname{Pre}\left(\delta_{n, k}^{\prime}\right) \notin K$. Then, by Proposition 3, $\delta_{n+N, i} \rightarrow$ $\neg \operatorname{Pre}\left(\delta_{n, k}^{\prime}\right) \in K$. Let $\delta_{0}^{\prime}$ be the propositional part of $\delta_{n, k}^{\prime}$. Then, by axiom $A_{6}$, $\neg \operatorname{Pre}\left(\delta_{0}^{\prime}\right), \delta_{0}^{\prime} \vdash \perp$, i.e. $\neg \operatorname{Pre}\left(\delta_{n, k}^{\prime}\right), \delta_{0}^{\prime} \vdash \perp$. Therefore, by rules $R_{9}$ and $R_{10}$ and because $\delta_{n, k}^{\prime} \rightarrow \delta_{0}^{\prime} \in K^{\prime}, \delta_{n+N, i} \rightarrow \neg \operatorname{Pre}\left(\delta_{n, k}^{\prime}\right) \in K$ and $\perp \rightarrow \bigvee_{l \in I^{\prime \prime}} \delta_{n, l}^{\prime \prime} \in K$, we obtain that $\delta_{n+N, i}, \delta_{n, k}^{\prime} \vdash \bigvee_{l \in I^{\prime \prime}} \delta_{n, l}^{\prime \prime}$.

(b) Second, assume that $\delta_{n+N, i} \rightarrow \operatorname{Pre}\left(\delta_{n, k}^{\prime}\right) \in K$. Assume towards a contradiction that $\delta_{n+N, i}, \delta_{n, k}^{\prime} \vdash \bigvee_{l \in I^{\prime \prime}} \delta_{n, l}^{\prime \prime}$ does not hold. We are going to show that $\delta_{n+N, i}, \delta_{n, k}^{\prime} \models \bigvee_{l \in I^{\prime \prime}} \delta_{n, l}^{\prime \prime}$ does not hold, contradicting $(*)$. By our assumption, for all $l \in I^{\prime \prime}, \delta_{n+N, i}, \delta_{n, k}^{\prime} \vdash \delta_{n, l}^{\prime \prime}$ does not hold. Hence, by application of Proposition 13, and because $\delta_{n+N, i} \rightarrow \operatorname{Pre}\left(\delta_{n, k}^{\prime}\right) \in K, \delta_{n+N, i}, \delta_{n, k}^{\prime} \models \delta_{n, l}^{\prime \prime}$ does not hold for all $l \in I^{\prime \prime}$. Then, by application of Lemma $12, \delta_{n+N, i}, \delta_{n, k}^{\prime} \models \neg \delta_{n, l}^{\prime \prime}$ for all $l \in I^{\prime \prime}$. Hence, $\delta_{n+N, i}, \delta_{n, k}^{\prime} \models \neg \bigvee_{l \in I^{\prime \prime}} \delta_{n, l}^{\prime \prime}$. Therefore, still by application of Lemma 12 and because $\delta_{n+N, i} \rightarrow \operatorname{Pre}\left(\delta_{n, k}^{\prime}\right) \in K$, we have that $\delta_{n+N, i}, \delta_{n, k}^{\prime} \models \bigvee_{l \in I^{\prime \prime}} \delta_{n, l}^{\prime \prime}$. This contradicts $(*)$.

So, we have proved that in both cases, for all $i \in I$ and $k \in I^{\prime}, \delta_{n+N, i}, \delta_{n, k}^{\prime} \vdash \bigvee_{l \in I^{\prime \prime}} \delta_{n, l}^{\prime \prime}$. Then, by rules $R_{8}$ and $R_{7}, \bigvee_{i \in I} \delta_{n+N, i}, \bigvee_{k \in I^{\prime}} \delta_{n, k}^{\prime} \vdash \bigvee_{l \in I^{\prime \prime}} \delta_{n, l}^{\prime \prime}$. Finally, by rules $R_{9}$ and $R_{11}$, we obtain that $\phi, \phi^{\prime} \vdash \phi^{\prime \prime}$.

Theorem 15. [Aucher et al., 2011] Given some formulas $\phi, \phi^{\prime \prime} \in \mathcal{L}$ and $\phi^{\prime} \in \mathcal{L}^{\prime}$, the problem of determining whether $\phi, \phi^{\prime} \models \phi^{\prime \prime}$ holds is decidable and NEXPTIMEcomplete.

The completeness result can be used to show that the Sequent Calculus SC and $\mathrm{SC}^{*}$ are provably equivalent.

Proposition 16. Let $\phi, \phi^{\prime \prime} \in \mathcal{L}$ and $\phi^{\prime} \in \mathcal{L}^{\prime}$. It holds that $\phi, \phi^{\prime} \vdash \phi^{\prime \prime}$ if and only if $\phi, \phi^{\prime} \vdash^{*} \phi^{\prime \prime}$.

Proof. By Lemma 10, we have that $\phi,\left.\phi^{\prime}\right|^{*} \phi^{\prime \prime}$ implies $\phi, \phi^{\prime} \vdash \phi^{\prime \prime}$. To prove the second direction, i.e. $\phi, \phi^{\prime} \vdash \phi^{\prime \prime}$ implies $\phi, \phi^{\prime} \vdash^{*} \phi^{\prime \prime}$, it suffices to observe that in the proof of Theorem 14, we only use the axiom schemata and rules of SC*. Therefore, if $\phi, \phi^{\prime} \models \phi^{\prime \prime}$, then $\phi, \phi^{\prime} \vdash^{*} \phi^{\prime \prime}$. Now, by soundness of SC, we have that $\phi, \phi^{\prime} \vdash \phi^{\prime \prime}$ implies $\phi, \phi^{\prime} \models \phi^{\prime \prime}$. Hence, $\phi, \phi^{\prime} \vdash \phi^{\prime \prime}$ implies $\phi, \phi^{\prime} \vdash^{*} \phi^{\prime \prime}$. 


\section{Progression of $\phi$ by $\phi^{\prime}: \phi \otimes \phi^{\prime}$}

The axiomatization of $\phi, \phi^{\prime} \vdash \phi^{\prime \prime}$ of Definition 15 provides us with a means to compute all the consequences $\phi^{\prime \prime}$ about a situation resulting from the occurrence of an event described by $\phi^{\prime}$ in an initial situation described by $\phi$. However, even if this axiomatization stays close to the definition of the update product and therefore provides a natural characterization of updates, we could wonder if there is a more compact way to represent all these consequences. This is what we will show in this section by introducing the notion of progression of $\phi$ by $\phi^{\prime}$.

Definition 17 (Progression of $\phi$ by $\phi^{\prime}, \phi \otimes \phi^{\prime}$ ). Let $\phi, \phi^{\prime \prime} \in \mathcal{L}$ and $\phi^{\prime} \in \mathcal{L}^{\prime}$. Let $n=\max \left\{\operatorname{deg}(\phi), \operatorname{deg}\left(\phi^{\prime}\right)\right\}, N=\max \left\{\operatorname{deg}\left(\operatorname{Pre}\left(p^{\prime}\right)\right) \mid p^{\prime} \in P^{\prime}\left(\phi^{\prime}\right)\right\}$ and $P=P(\phi) \cup$ $\bigcup\left\{P\left(\operatorname{Pre}\left(p^{\prime}\right)\right) \mid p^{\prime} \in P^{\prime}\left(\phi^{\prime}\right)\right\}$. Then, by Corollaries 1 and 2, there are $S \subseteq S_{n+N}^{P}$ and $S^{\prime} \subseteq S_{n}^{P^{\prime}}$ such that $\phi \leftrightarrow \bigvee_{\delta \in S} \delta \in \mathrm{K}$ and $\phi^{\prime} \leftrightarrow \bigvee_{\delta^{\prime} \in S^{\prime}} \delta^{\prime} \in \mathrm{K}^{\prime}$. We define the progression of $\phi$ by $\phi^{\prime}$, written $\phi \otimes \phi^{\prime}$, as follows:

$$
\phi \otimes \phi^{\prime}=\bigvee\left\{\delta \otimes \delta^{\prime} \mid\left(\delta, \delta^{\prime}\right) \in S \otimes S^{\prime}\right\}
$$

where $S \otimes S^{\prime}=\left\{\left(\delta, \delta^{\prime}\right) \in S \times S^{\prime} \mid \delta \rightarrow \operatorname{Pr} e\left(\delta^{\prime}\right) \in \mathrm{K}\right\}$ and for all $\left(\delta, \delta^{\prime}\right) \in S \otimes S^{\prime}, \delta \otimes \delta^{\prime}$ is defined inductively as follows:

$\mathbf{n}=\mathbf{0}$ :

$$
\delta \otimes \delta^{\prime}=\bigvee_{\delta^{\prime \prime} \in \operatorname{Ref}(\delta)} \delta^{\prime \prime}
$$

$$
\begin{aligned}
& \text { n+1: If } \delta=\delta_{0} \wedge \bigwedge_{j \in A g t}\left(\bigwedge_{\delta_{j} \in R_{j}(\delta)}\left\langle B_{j}\right\rangle \delta_{j} \wedge B_{j}\left(\bigvee_{\delta_{j} \in R_{j}(\delta)} \delta_{j}\right)\right) \\
& \delta^{\prime}=\delta_{0}^{\prime} \wedge \bigwedge_{j \in A g t}\left(\bigwedge_{\delta_{j}^{\prime} \in R_{j}\left(\delta^{\prime}\right)}\left\langle B_{j}\right\rangle \delta_{j}^{\prime} \wedge B_{j}\left(\bigvee_{\delta_{j}^{\prime} \in R_{j}\left(\delta^{\prime}\right)} \delta_{j}^{\prime}\right)\right), \\
& \text { then } \delta \otimes \delta^{\prime}=\delta_{0} \wedge \bigwedge_{j \in A g t}\left(\bigwedge\left\{\left\langle B_{j}\right\rangle\left(\delta_{j} \otimes \delta_{j}^{\prime}\right) \mid\left(\delta_{j}, \delta_{j}^{\prime}\right) \in R_{j}(\delta) \otimes R_{j}\left(\delta^{\prime}\right)\right\}\right. \\
& \left.\wedge B_{j}\left(\bigvee\left\{\delta_{j} \otimes \delta_{j}^{\prime} \mid\left(\delta_{j}, \delta_{j}^{\prime}\right) \in R_{j}(\delta) \otimes R_{j}\left(\delta^{\prime}\right)\right\}\right)\right)
\end{aligned}
$$

where $R_{j}(\delta) \otimes R_{j}\left(\delta^{\prime}\right)=\left\{\left(\delta_{j}, \delta_{j}^{\prime}\right) \in R_{j}(\delta) \times R_{j}\left(\delta^{\prime}\right) \mid \delta_{j} \rightarrow \operatorname{Pre}\left(\delta_{j}^{\prime}\right) \in \mathrm{K}\right\}$.

Note that if $\delta$ and $\delta^{\prime}$ represent syntactically a pointed $\mathcal{L}$-model $(M, w)$ and a pointed $\mathcal{L}^{\prime}$-model $\left(M^{\prime}, w^{\prime}\right)$ respectively, then the way the formula $\delta \otimes \delta^{\prime}$ is defined is a syntactic counterpart of the definition of the product update $\otimes$ of $(M, w)$ by $\left(M^{\prime}, w^{\prime}\right)$. 
Theorem 17. Let $\phi, \phi^{\prime \prime} \in \mathcal{L}$ and $\phi^{\prime} \in \mathcal{L}^{\prime}$. It holds that $\phi, \phi^{\prime} \models \phi^{\prime \prime}$ if and only if $\phi \otimes \phi^{\prime} \rightarrow \phi^{\prime \prime} \in K$.

As the above theorem shows, progression is an analogue in a dynamic setting of the notion of prime implicate. In propositional logic, $\lambda$ is a prime implicate of $\phi$ if $\phi \vdash \lambda$ and for all $\lambda^{\prime}$ such that $\phi \vdash \lambda^{\prime}$, if $\lambda^{\prime} \vdash \lambda$ then $\lambda \vdash \lambda^{\prime}$ (a similar notion has also been defined in first-order logic and modal logic). Our operator $\otimes$ has also the same import as the operator $\otimes$ of [Baltag et al., 2005], although it is used there in a different, i.e. algebraic, setting. To prove Theorem 17, we first prove two lemmas:

Lemma 18. Let $P^{\prime}$ be a finite subset of $\Phi^{\prime}$ and let $P$ be a finite subset of $\Phi$ such that $\bigcup\left\{P\left(\operatorname{Pre}\left(p^{\prime}\right)\right) \mid p^{\prime} \in P^{\prime}\right\} \subseteq P$. Let $n \in \mathbb{N}$ and $N=\max \left\{\operatorname{deg}\left(\operatorname{Pre}\left(p^{\prime}\right)\right) \mid p^{\prime} \in P^{\prime}\right\}$. Let $\delta \in S_{n+N}^{P}$ and $\delta^{\prime} \in S_{n}^{P^{\prime}}$. Let $(M, w)$ be a pointed $\mathcal{L}$-model and $\left(M^{\prime}, w^{\prime}\right)$ be a pointed $\mathcal{L}^{\prime}$ model such that $M, w \vDash \operatorname{Pre}\left(w^{\prime}\right), M, w \vDash \delta$ and $M^{\prime}, w^{\prime} \vDash \delta^{\prime}$. Then $(M, w) \otimes\left(M^{\prime}, w^{\prime}\right) \vDash$ $\delta \otimes \delta^{\prime}$.

Proof. By induction on $n$.

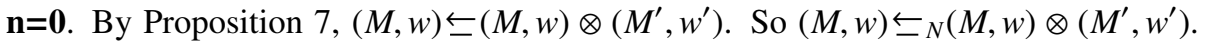
Then $(M, w) \otimes\left(M^{\prime}, w^{\prime}\right) \vDash \delta_{N}^{\prime \prime}$ for some $\delta_{N}^{\prime \prime} \in \operatorname{Re} f\left(\delta_{N}\right)$ by Proposition 8 . So, by definition of $\delta \otimes \delta^{\prime},(M, w) \otimes\left(M^{\prime}, w^{\prime}\right) \vDash \delta \otimes \delta^{\prime}$.

n+1. Assume that $M, w \vDash \delta$ and $M^{\prime}, w^{\prime} \vDash \delta^{\prime}$ for some $\delta \in S_{n+1+N}^{P}$ and $\delta^{\prime} \in S_{n+1}^{P^{\prime}}$.

$$
\begin{aligned}
& \delta=\delta_{0} \wedge \bigwedge_{j \in A g t}\left(\bigwedge_{\delta_{j} \in R_{j}(\delta)}\left\langle B_{j}\right\rangle \delta_{n} \wedge B_{j}\left(\bigvee_{\delta_{j} \in R_{j}(\delta)} \delta_{n+N}\right)\right), \\
& \delta^{\prime}=\delta_{0}^{\prime} \wedge \bigwedge_{j \in A g t}\left(\bigwedge_{\delta_{j}^{\prime} \in R_{j}\left(\delta^{\prime}\right)}\left\langle B_{j}\right\rangle \delta_{j}^{\prime} \wedge B_{j}\left(\bigvee_{\delta_{j}^{\prime} \in R_{j}\left(\delta^{\prime}\right)} \delta_{j}^{\prime}\right)\right) .
\end{aligned}
$$

Let $\left(M^{\prime \prime},\left(w, w^{\prime}\right)\right)=(M, w) \otimes\left(M^{\prime}, w^{\prime}\right)$ and $\left(v, v^{\prime}\right) \in R_{j}\left(w, w^{\prime}\right)$. Then $v \in R_{j}(w), v^{\prime} \in$ $R_{j}\left(w^{\prime}\right)$ and $M, v \vDash \operatorname{Pre}\left(v^{\prime}\right)$. Then, $M, v \vDash \delta_{j}$ for some $\delta_{j} \in R_{j}(\delta), M^{\prime}, v^{\prime} \vDash \delta_{j}^{\prime}$ for some $\delta_{j}^{\prime} \in R_{j}\left(\delta^{\prime}\right)$ and $\delta_{j} \rightarrow \operatorname{Pre}\left(\delta_{j}^{\prime}\right) \in \mathrm{K}$. Then, by induction hypothesis, $M^{\prime \prime},\left(v, v^{\prime}\right) \vDash \delta_{j} \otimes \delta_{j}^{\prime}$.

Reciprocally, for all $\delta_{j} \in R_{j}(\delta)$ and $\delta_{j}^{\prime} \in R_{j}\left(\delta^{\prime}\right)$ such that $\delta_{j} \rightarrow \operatorname{Pre}\left(\delta_{j}^{\prime}\right) \in \mathrm{K}$, there is $\left(v, v^{\prime}\right) \in R_{j}\left(w, w^{\prime}\right)$ such that $M, v \vDash \delta_{j}, M^{\prime}, v^{\prime} \vDash \delta_{j}^{\prime}$, and therefore also $M^{\prime \prime},\left(v, v^{\prime}\right) \vDash \delta_{j} \otimes \delta_{j}^{\prime}$ by induction hypothesis. So:

$$
\begin{aligned}
(M, w) \otimes\left(M^{\prime}, w^{\prime}\right)=\delta_{0} \wedge \bigwedge_{j \in A g t} & \wedge\left\{\left\langle B_{j}\right\rangle\left(\delta_{j} \otimes \delta_{j}^{\prime}\right) \mid\left(\delta_{j}, \delta_{j}^{\prime}\right) \in R_{j}(\delta) \otimes R_{j}\left(\delta^{\prime}\right)\right\} \\
\wedge & \left.B_{j}\left(\bigvee\left\{\delta_{j} \otimes \delta_{j}^{\prime} \mid\left(\delta_{j}, \delta_{j}^{\prime}\right) \in R_{j}(\delta) \otimes R_{j}\left(\delta^{\prime}\right)\right\}\right)\right)
\end{aligned}
$$

i.e. $(M, w) \otimes\left(M^{\prime}, w^{\prime}\right) \vDash \delta \otimes \delta^{\prime}$.

Lemma 19. Let $n \in \mathbb{N}$, let $P^{\prime}$ be a finite subset of $\Phi^{\prime}$ and let $P$ be a finite subset of $\Phi$ such that $\bigcup\left\{P\left(\operatorname{Pre}\left(p^{\prime}\right)\right) \mid p^{\prime} \in P^{\prime}\right\} \subseteq P$. Let $\delta \in S_{n+N}^{P}$ and $\delta^{\prime} \in S_{n}^{P^{\prime}}$ such that 
$\delta \rightarrow \operatorname{Pre}\left(\delta^{\prime}\right) \in K$. Let $\left(M^{\prime \prime}, w^{\prime \prime}\right)$ be a finite and pointed $\mathcal{L}$-model. If $M^{\prime \prime}, w^{\prime \prime} \vDash \delta \otimes \delta^{\prime}$, then there is a pointed $\mathcal{L}$-model $(M, w)$ and a pointed $\mathcal{L}^{\prime}$-model $\left(M^{\prime}, w^{\prime}\right)$ such that $\left(M^{\prime \prime}, w^{\prime \prime}\right) \leftrightarrow(M, w) \otimes\left(M^{\prime}, w^{\prime}\right), M, w \vDash \delta$ and $M^{\prime}, w^{\prime} \vDash \delta^{\prime}$.

Proof. By induction on $n$.

$\mathbf{n}=\mathbf{0}$. Let $\left(M^{\prime \prime}, w^{\prime \prime}\right)$ be a finite pointed $\mathcal{L}$-model and assume that $M^{\prime \prime}, w^{\prime \prime} \vDash \delta \otimes \delta^{\prime}$ for some $\delta \in S_{N}^{P}$ and $\delta^{\prime} \in S_{0}^{P^{\prime}}$. Then, $M^{\prime \prime}, w^{\prime \prime} \vDash \bigvee_{\delta^{\prime \prime} \in \operatorname{Ref}(\delta)} \delta^{\prime \prime}$ by definition of $\delta \otimes \delta^{\prime}$.

Let $(M, w)$ be a finite and pointed $\mathcal{L}$-model such that the modal structure of $(M, w)$ from depth $N$ on is the same as $\left(M^{\prime \prime}, w^{\prime \prime}\right)$ and such that $M, w \vDash \delta$. Then

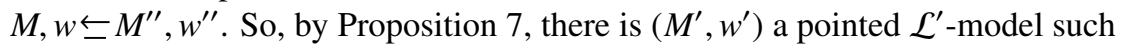
that $\left(M^{\prime \prime}, w^{\prime \prime}\right)=(M, w) \otimes\left(M^{\prime}, w^{\prime}\right)$. Moreover, $M^{\prime}, w^{\prime} \vDash \delta^{\prime}$ because $\operatorname{deg}\left(\delta^{\prime}\right)=0$.

n + 1. Let $\left(M^{\prime \prime}, w^{\prime \prime}\right)$ be a pointed $\mathcal{L}$-model such that $M^{\prime \prime}, w^{\prime \prime} \vDash \delta \otimes \delta^{\prime}$ for some $\delta \in S_{n+N}^{P}$ and $\delta^{\prime} \in S_{n}^{P^{\prime}}$. Then, for all $v^{\prime \prime} \in R_{j}\left(w^{\prime \prime}\right)$, there are $\left(\delta_{j}, \delta_{j}^{\prime}\right) \in R_{j}(\delta) \otimes R_{j}\left(\delta^{\prime}\right)$ such that $M^{\prime \prime}, v^{\prime \prime} \vDash \delta_{j} \otimes \delta_{j}^{\prime}$. Consequently, by induction hypothesis, for all $v^{\prime \prime} \in$ $R_{j}\left(w^{\prime \prime}\right)$, there are a finite and pointed $\mathcal{L}$-model $\left(M_{j, v^{\prime \prime}}, v_{j, v^{\prime \prime}}\right)$ and a pointed $\mathcal{L}^{\prime}$ model $\left(M_{j, v^{\prime \prime}}^{\prime}, v_{j, v^{\prime \prime}}^{\prime}\right)$ such that $\left(M^{\prime \prime}, v^{\prime \prime}\right)=\left(M_{j, v^{\prime \prime}}, v_{j, v^{\prime \prime}}\right) \otimes\left(M_{j, v^{\prime \prime}}^{\prime}, v_{j, v^{\prime \prime}}^{\prime}\right), M, v \vDash \delta_{j}$ and $M^{\prime}, v^{\prime} \vDash \delta_{j}^{\prime}$.

We then build a $\mathcal{L}$-model $(M, w)$ by introducing a new world $w$ with valuation $\delta_{0}$ (the atomic facts not in $P$ are set to false in this possible world) and by linking this world $w$ to the pointed $\mathcal{L}$-model $\left(M_{j, v^{\prime \prime}}, v_{j, v^{\prime \prime}}\right)$ by an accessibility relation indexed by $j$, and so for all $v^{\prime \prime} \in R_{j}\left(w^{\prime \prime}\right)$ and all $j \in A g t$. We also link world $w$ to pointed $\mathcal{L}$-models $\left(M_{\delta_{j}}, w_{\delta_{j}}\right)$ such that $M_{\delta_{j}}, w_{\delta_{j}} \models \delta_{j}$, and so for the remaining $\delta_{j} \mathrm{~s}$ in $R_{j}(\delta)$. We build similarly a $\mathcal{L}^{\prime}$-model $\left(M^{\prime}, w^{\prime}\right)$ by introducing a new possible event $w^{\prime}$ with valuation $\delta_{0}^{\prime}$ (the atomic events not in $P^{\prime}$ are set to false in this possible event) and by linking this new possible event $w^{\prime}$ to the pointed $\mathcal{L}^{\prime}$-model $\left(M_{j, v^{\prime \prime}}^{\prime}, v_{j, v^{\prime \prime}}^{\prime}\right)$ by an accessibility relation indexed by $j$, and so for all $v^{\prime \prime} \in R_{j}\left(w^{\prime \prime}\right)$ and all $j \in A g t$. We also link world $w^{\prime}$ to pointed $\mathcal{L}^{\prime}$-models $\left(M_{\delta_{j}}^{\prime}, w_{\delta_{j}^{\prime}}^{\prime}\right)$ such that $M_{\delta_{j}}^{\prime}, w_{\delta_{j}^{\prime}}^{\prime}=\delta_{j}^{\prime}$, and so for the remaining $\delta_{j}^{\prime} \mathrm{s}$ in $R_{j}\left(\delta^{\prime}\right)$. One can then easily show

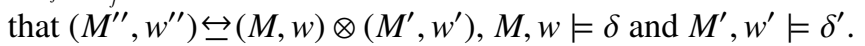

We can now prove Theorem 17:

Proof (of Theorem 17). We first prove that for all $n \in \mathbb{N}$, all $\delta \in S_{n}^{P}$ and all $\delta^{\prime} \in S_{n}^{P^{\prime}}$, we have that $\delta \otimes \delta^{\prime} \rightarrow \phi^{\prime \prime} \in \mathrm{K}$ iff $\delta, \delta^{\prime} \vdash \phi^{\prime \prime}$.

- Assume that $\delta \otimes \delta^{\prime} \rightarrow \phi^{\prime \prime} \in \mathrm{K}$. Let $(M, w)$ be a pointed $\mathcal{L}$-model and $\left(M^{\prime}, w^{\prime}\right)$ be a pointed $\mathcal{L}$-model such that $M, w \vDash \operatorname{Pre}\left(w^{\prime}\right), M, w \vDash \delta$ and $M^{\prime}, w^{\prime} \vDash \delta^{\prime}$. Then, by Lemma $18,(M, w) \otimes\left(M^{\prime}, w^{\prime}\right) \vDash \delta \otimes \delta^{\prime}$. So $(M, w) \otimes\left(M^{\prime}, w^{\prime}\right) \vDash \phi^{\prime \prime}$ by assumption. Then, $\delta, \delta^{\prime} \vdash \phi^{\prime \prime}$.

- Assume that $\delta, \delta^{\prime} \vdash \phi^{\prime \prime}$. If $\delta \otimes \delta^{\prime}$ is $\mathrm{K}$-inconsistent, then the result trivially holds. Otherwise, let $\left(M^{\prime \prime}, w^{\prime \prime}\right)$ be a finite $\mathcal{L}$-model such that $M^{\prime \prime}, w^{\prime \prime} \vDash \delta \otimes \delta^{\prime}$. Note that because $\mathrm{K}$ has the finite model property with respect to the class of $\mathcal{L}$-models, such a model exists. Then, by Lemma 19 , there is a pointed $\mathcal{L}$-model $(M, w)$ and a pointed $\mathcal{L}^{\prime}$-model $\left(M^{\prime}, w^{\prime}\right)$ such that $\left(M^{\prime \prime}, w^{\prime \prime}\right) \stackrel{\leftrightarrow}{-}(M, w) \otimes\left(M^{\prime}, w^{\prime}\right)$ and 
$M, w \vDash \delta, M^{\prime}, w^{\prime} \vDash \delta^{\prime}$. So $M^{\prime \prime}, w^{\prime \prime} \vDash \phi^{\prime \prime}$ by assumption. Therefore, for all finite $\mathcal{L}$-model $M$, it holds that $M \vDash \delta \otimes \delta \rightarrow \phi^{\prime \prime}$. Hence, because $\mathrm{K}$ has the finite model property with respect to the class of $\mathcal{L}$-models, $\delta \otimes \delta \rightarrow \phi^{\prime \prime} \in \mathrm{K}$.

Let $\phi, \phi^{\prime \prime} \in \mathcal{L}$ and $\phi^{\prime} \in \mathcal{L}^{\prime}$. Let $n=\max \left\{\operatorname{deg}(\phi), \operatorname{deg}\left(\phi^{\prime}\right)\right\}$ and $N=\max \left\{\operatorname{Pre}\left(p^{\prime}\right) \mid p^{\prime} \in P^{\prime}\right\}$. Then, by Corollaries 1 and 2, there are $S \subseteq S_{n+N}^{P}$ and $S^{\prime} \subseteq S_{n}^{P^{\prime}}$ such that $\phi \leftrightarrow \bigvee_{\delta \in S} \delta \in \mathrm{K}$ and $\phi^{\prime} \leftrightarrow \bigvee \delta^{\prime} \in \mathrm{K}^{\prime}$. Therefore, $\phi, \phi^{\prime} \vdash \phi^{\prime \prime}$

iff $\bigvee_{\delta \in S} \delta, \bigvee_{\delta^{\prime} \in S^{\prime}}^{\delta^{\prime} \in S^{\prime}} \delta^{\prime} \vdash \phi^{\prime \prime}$ by rule $\mathrm{R}_{9}$ and $\mathrm{R}_{10}$

iff for all $\delta \in S$, all $\delta^{\prime} \in S^{\prime}$, we have that $\delta, \delta^{\prime} \vdash \phi^{\prime \prime}$ by rules $\mathrm{R}_{7}$ and $\mathrm{R}_{8}$ iff for all $\delta \in S$, all $\delta^{\prime} \in S^{\prime}$, we have that $\delta \otimes \delta^{\prime} \rightarrow \phi^{\prime \prime} \in \mathrm{K}$

iff $\bigvee_{\delta S, \delta^{\prime}, S^{\prime}} \delta \otimes \delta^{\prime} \rightarrow \phi^{\prime \prime} \in \mathrm{K}$

iff $\stackrel{\delta \in S, \delta^{\prime}, S^{\prime}}{\phi \otimes} \phi^{\prime} \rightarrow \phi^{\prime \prime} \in \mathrm{K}$.

\section{Extension of our results to other modal logics}

In this section, we show how our results extend to other logics than $\mathrm{K}_{\text {and }} \mathrm{K}^{\prime}$, under the condition that the class of models they define is stable for the product update.

Let $C$ be a class of $\mathcal{L}$-models and $C^{\prime}$ be a class of $\mathcal{L}^{\prime}$-models. $C$ is stable for the product update with respect to the class $C^{\prime}$ when for all $M \in C$ and all $M^{\prime} \in C^{\prime}$, for all $w \in M$ and all $w^{\prime} \in M^{\prime}$ such that $M, w \vDash \operatorname{Pre}\left(w^{\prime}\right),(M, w) \otimes\left(M^{\prime}, w^{\prime}\right)$ is a pointed $\mathcal{L}$-model of $C$. As noted in [van Benthem, 2007], the only first-order frame conditions that are stable for the product update are those definable as universal Horn sentences. Reflexivity, symmetry, and transitivity are of this special form.

Let $C$ be a class of $\mathcal{L}$-models and let $C^{\prime}$ be a class of $\mathcal{L}^{\prime}$-models. The inference relation $\phi,\left.\phi^{\prime}\right|_{\overline{C, C^{\prime}}} \phi^{\prime \prime}$ is defined as follows:

$\phi, \phi^{\prime} \varlimsup_{\overline{C, C^{\prime}}} \phi^{\prime \prime} \quad$ iff for all pointed $\mathcal{L}$-model $(M, w)$ of $C$, and $\mathcal{L}^{\prime}$-model $\left(M^{\prime}, w^{\prime}\right)$ of $C^{\prime}$ such that $M, w \models \operatorname{Pre}\left(w^{\prime}\right)$, if $M, w \vDash \phi$ and $M^{\prime}, w^{\prime} \vDash \phi^{\prime}$ then $(M, w) \otimes\left(M^{\prime}, w^{\prime}\right) \vDash \phi^{\prime \prime}$

Let $L$ be a logic for the language $\mathcal{L}$ containing the logic $K$ and let $L^{\prime}$ be a logic for the language $\mathcal{L}^{\prime}$ containing the logic $\mathrm{K}^{\prime}$. The DEL-sequent calculus $S C_{L, L^{\prime}}$ is defined as the DEL-sequent calculus SC, except that the logic $\mathrm{K}$ (resp. $\mathrm{K}^{\prime}$ ) is replaced by the $\operatorname{logic} \mathrm{L}$ (resp. $\mathrm{L}^{\prime}$ ). A DEL-sequent of $\mathrm{SC}_{\mathrm{L}, \mathrm{L}^{\prime}}$ is written $\phi,\left.\phi^{\prime}\right|_{\mathrm{L}, \mathrm{L}^{\prime}} \phi^{\prime \prime}$.

Theorem 20. Let $L$ be a logic sound and complete for $\mathcal{L}$ with respect to a class $C$ of $\mathcal{L}$-models and let $L^{\prime}$ be a logic sound and complete for $\mathcal{L}^{\prime}$ with respect to a class $C^{\prime}$ of $\mathcal{L}^{\prime}$-models. If $C$ is stable for the product update with respect to the class $C^{\prime}$, then for all $\phi, \phi^{\prime \prime} \in \mathcal{L}$ and all $\phi^{\prime} \in \mathcal{L}^{\prime}$, it holds that $\phi,\left.\phi^{\prime}\right|_{\overline{C, C^{\prime}}} \phi^{\prime \prime}$ if and only if $\phi,\left.\phi^{\prime}\right|_{L, L^{\prime}} \phi^{\prime \prime}$.

Proof. It can easily be adapted from the proofs of Theorems 9 and 14 . 


\section{Example}

Let us take up our card game example of Section 3. We formalize the general setting of the game in a logic $L$ defined as follows:

Definition 18 (Logic $\mathrm{L}$ ). The logic $\mathrm{L}$ is defined by adding to the logic $\mathrm{K}$ the following axiom schemata, where $j$ ranges over $\{A, B, C\}$ and $\operatorname{Card}$ over $\{r, w, b\}$.

$$
\begin{array}{ll}
(\text { Card Game CG1) } & \vdash \operatorname{Card}_{j} \rightarrow \neg \operatorname{Card}_{i} \quad \text { for all } i \neq j \\
(\text { Card Game CG2) } & \vdash r_{j} \vee b_{j} \vee w_{j} \\
\text { (Card Game CG3) } & \vdash \operatorname{Card}_{j} \rightarrow B_{j} \operatorname{Card}_{j}
\end{array}
$$

Axioms $C G 1$ and $C G 2$ state that players $\mathrm{A}, \mathrm{B}, \mathrm{C}$ have a unique card. Axiom $C G 3$ states that the players 'know' which card they have. Then we can derive in the DELsequent calculus $\mathrm{SC}_{\mathrm{L}, \mathrm{K}^{\prime}}$ the following statements. We recall that the atomic event $p^{\prime}$ stands for 'player A shows her red card'.

$$
\mathrm{\top},\left.B_{j} p^{\prime}\right|_{\left\llcorner, \mathrm{K}^{\prime}\right.} B_{j} r_{A} \quad \text { (Inference 1) } \quad w_{B},\left.B_{B} p^{\prime}\right|_{\left\llcorner, \mathrm{K}^{\prime}\right.} B_{B} b_{C} \quad \text { (Inference 2) }
$$

Inference 1 states that player $j$ believes that player A has the red card after any event during which player $j$ believed that player A showed her red card. Inference 2 states that if player B has the white card, then after an event during which he believes that player A shows her red card, he believes that player $\mathrm{C}$ has the blue card. We provide below the proof of these two inferences.

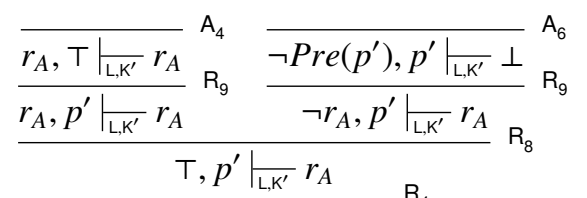

$$
\begin{aligned}
& \frac{\frac{\mathrm{T} p p_{\mathrm{L}, \mathrm{K}^{\prime}}}{B_{j} \mathrm{~T},\left.B_{j} p^{\prime}\right|_{\left\llcorner, \mathrm{K}^{\prime}\right.}} B_{j} r_{A}}{\mathrm{~T},\left.B_{j} p^{\prime}\right|_{L_{\mathrm{L}, K^{\prime}}} B_{j} r_{A}} \mathrm{R}_{4}{ }_{\mathrm{T} \rightarrow B_{j} \mathrm{~T} \in \mathrm{K}, \mathrm{R}_{9}}
\end{aligned}
$$

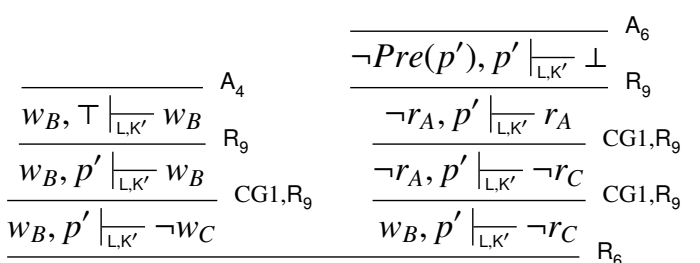

$$
\begin{aligned}
& w_{B},\left.p^{\prime}\right|_{L, \mathrm{~K}^{\prime}} \neg w_{C} \wedge \neg r_{C}{ }_{\mathrm{CG} 2, \mathrm{R}_{9}}
\end{aligned}
$$

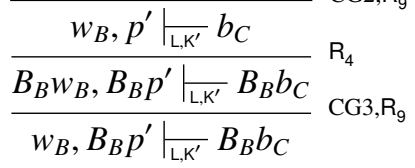




\section{Concluding remarks}

Even if our axiomatizations provide a natural characterization of the standard BMS product update, as we argued in the comments following Definition 15, it still remains to show that our approach can be adapted to the analysis of other product updates. If one considers product updates defined on the basis of a relational Kripkestyle semantics, as in this paper, one has to admit that few alternatives to the product update of Definition 8 exist in the DEL literature ([Liu, 2008] mentions some of them) and that eliciting a large family of new and meaningful product updates seems at first sight difficult. On the other hand, if the logical framework presented in this paper is enriched with more refined representations of uncertainty (by means of plausibility or probability measures for instance), other kinds of product updates can be studied, which address other kinds of dynamics, such as belief revision or nonmonotonic reasoning. As it turns out, numerous product update rules for belief revision have been proposed in this richer setting [Aucher, 2004, van Ditmarsch, 2005, Aucher, 2007, Baltag \& Smets, 2006, Baltag \& Smets, 2008, van Benthem, 2007, Liu, 2008, van Benthem et al., 2009b]. Nevertheless, one still needs to show that the proof techniques developped in this paper can be adapted to these more refined representations of uncertainty.

Acknowledgements. I thank Alexandru Baltag for helpful comments on a preliminary version of this paper [Aucher, 2010]. I also thank two anonymous referees for their extensive and very useful reviews which prompted me to rewrite the paper entirely.

\section{References}

[Alchourrón et al., 1985] Alchourrón, C., Gärdenfors, P., \& Makinson, D. (1985). On the logic of theory change: Partial meet contraction and revision functions. Journal of Symbolic Logic, 50(2), 510-530.

[Aucher, 2004] Aucher, G. (2004). A combined system for update logic and belief revision. In M. Barley \& N. K. Kasabov (Eds.), PRIMA, volume 3371 of Lecture Notes in Computer Science (pp. 1-17).: Springer.

[Aucher, 2007] Aucher, G. (2007). Interpreting an action from what we perceive and what we expect. Journal of Applied Non-Classical Logics, 17(1), 9-38.

[Aucher, 2009] Aucher, G. (2009). BMS revisited. In A. Heifetz (Ed.), TARK (pp. 24-33).

[Aucher, 2010] Aucher, G. (2010). Characterizing updates in dynamic epistemic logic. In F. Lin, U. Sattler, \& M. Truszczynski (Eds.), KR: AAAI Press.

[Aucher, 2012] Aucher, G. (2012). DEL-sequents for regression and epistemic planning. Journal of Applied Non-Classical Logics, forthcoming. 
[Aucher et al., 2011] Aucher, G., Maubert, B., \& Schwarzentruber, F. (2011). Tableau method and NEXPTIME-completeness of del-sequents. Electronic Notes in Theoretical Computer Science, 278, 17-30.

[Baltag et al., 2005] Baltag, A., Coecke, B., \& Sadrzadeh, M. (2005). Algebra and sequent calculus for epistemic actions. Electronic Notes in Theoretical Computer Science, 126, 27-52.

[Baltag et al., 2007] Baltag, A., Coecke, B., \& Sadrzadeh, M. (2007). Epistemic actions as resources. Journal of Logic and Computation, 17(3), 555-585.

[Baltag \& Moss, 2004] Baltag, A. \& Moss, L. (2004). Logic for epistemic programs. Synthese, 139(2), 165-224.

[Baltag et al., 1999] Baltag, A., Moss, L., \& Solecki, S. (1999). The Logic of Public Announcements, Common Knowledge and Private Suspicions. Technical report, Indiana University.

[Baltag \& Smets, 2006] Baltag, A. \& Smets, S. (2006). Conditional doxastic models: A qualitative approach to dynamic belief revision. Electronic Notes in Theoretical Computer Science, 165, 5-21.

[Baltag \& Smets, 2008] Baltag, A. \& Smets, S. (2008). Probabilistic dynamic belief revision. Synthese, 165(2), 179-202.

[van Benthem, 2003] van Benthem, J. (2003). Meaning: the Dynamic Turn, chapter Structural Properties of Dynamic Reasoning, (pp. 15-31). Elsevier: Amsterdam.

[van Benthem, 2007] van Benthem, J. (2007). Dynamic logic for belief revision. Journal of Applied Non-Classical Logics, 17(2), 129-155.

[van Benthem, 2011] van Benthem, J. (2011). McCarthy variations in a modal key. Artificial intelligence, 175(1), 428-439.

[van Benthem et al., 2009a] van Benthem, J., Gerbrandy, J., Hoshi, T., \& Pacuit, E. (2009a). Merging frameworks for interaction. Journal of Philosophical Logic, $38(5), 491-526$.

[van Benthem et al., 2009b] van Benthem, J., Gerbrandy, J., \& Kooi, B. (2009b). Dynamic update with probability. Studia Logica, 93(1), 67-96.

[van Benthem et al., 2006] van Benthem, J., van Eijck, J., \& Kooi, B. (2006). Logics of communication and change. Information and Computation, 204(11), 1620-1662.

[Blackburn et al., 2001] Blackburn, P., de Rijke, M., \& Venema, Y. (2001). Modal Logic, volume 53 of Cambridge Tracts in Computer Science. Cambridge University Press.

[Church, 1957] Church, A. (1957). Application of recursive arithmetic to the problem of circuit synthesis. In Summaries of the Summer Institute of Symbolic Logic, volume 1 (pp. 3-50). Cornell University. 
[Cordon-Franco et al., 2010] Cordon-Franco, A., van Ditmarsch, H., FernandezDuque, D., Gomez-Caminero, E., \& Nepomuceno-Fernandez, A. (2010). Two mischievous dynamic consequence relations. In X. Arrazola \& M. Ponte (Eds.), Int. Workshop on Logic and Philosophy of Knowledge, Communication and Action (pp. 197-208). Spain: University of the Basque Country Press.

[van Ditmarsch, 2005] van Ditmarsch, H. (2005). Prolegomena to dynamic logic for belief revision. Synthese, 147, 229-275.

[van Ditmarsch et al., 2007] van Ditmarsch, H., van der Hoek, W., \& Kooi, B. (2007). Dynamic Epistemic Logic, volume 337 of Synthese library. Springer.

[van Ditmarsch \& French, 2008] van Ditmarsch, H. P. \& French, T. (2008). Simulation and information: Quantifying over epistemic events. In J.-J. C. Meyer \& J. Broersen (Eds.), KRAMAS, volume 5605 of Lecture Notes in Computer Science (pp. 51-65).: Springer.

[van Ditmarsch et al., 2009] van Ditmarsch, H. P., Herzig, A., \& Lima, T. D. (2009). From situation calculus to dynamic epistemic logic. Journal of Logic and Computation, 21(2), 179-204.

[Fine, 1975] Fine, K. (1975). Normal forms in modal logic. Notre Dame Journal of Formal Logic, 16, 229-237.

[Halpern \& Moses, 1990] Halpern, J. \& Moses, Y. (1990). Knowledge and common knowledge in a distributed environment. Journal of the ACM, 37(3), 549-587.

[Hoare, 1969] Hoare, C. (1969). An axiomatic basis for computer programming. Communications of the ACM, 12(10), 567-580.

[Katsuno \& Mendelzon, 1992] Katsuno, H. \& Mendelzon, A. (1992). Propositional knowledge base revision and minimal change. Artificial Intelligence, 52(3), 263294.

[Kooi, 2007] Kooi, B. (2007). Expressivity and completeness for public update logics via reduction axioms. Journal of Applied Non-Classical Logics, 17(2), 231-253.

[Kupferman \& Vardi, 1999] Kupferman, O. \& Vardi, M. Y. (1999). Church's problem revisited. Bulletin of Symbolic Logic, 5(2), 245-263.

[Liu, 2008] Liu, F. (2008). Changing for the Better: Preference Dynamics and Agent Diversity. PhD thesis, ILLC, University of Amsterdam.

[van der Meyden \& Vardi, 1998] van der Meyden, R. \& Vardi, M. Y. (1998). Synthesis from knowledge-based specifications (extended abstract). In D. Sangiorgi \& R. de Simone (Eds.), CONCUR, volume 1466 of Lecture Notes in Computer Science (pp. 34-49).: Springer.

[Moss, 2007] Moss, L. S. (2007). Finite models constructed from canonical formulas. Journal of Philosophical Logic, 36(6), 605-640. 
[Pratt, 1976] Pratt, V. R. (1976). Semantical considerations on floyd-hoare logic. In FOCS (pp. 109-121).: IEEE Computer Society.

[Reiter, 2001] Reiter, R. (2001). Knowledge in Action: Logical Foundations for Specifying and Implementing Dynamical Systems. MIT Press.

[Shanahan, 1997] Shanahan, M. (1997). Solving the Frame Problem. Cambridge, Massachusetts: MIT press. 